\title{
Synthesis of medium ring heterocycles using an intramolecular Heck reaction
}

\author{
Leggy A. Arnold, Wenchen Luo, R. Kiplin Guy* \\ Departments of Pharmaceutical Chemistry and Cellular and \\ Molecular Pharmacology, University of California San Francisco, \\ $60016^{\text {th }}$ Street, San Francisco, CA 94143-2280, USA \\ rguy@cgl.ucsf.edu
}

\section{Supporting information}

\section{Materials described in Scheme 1.}
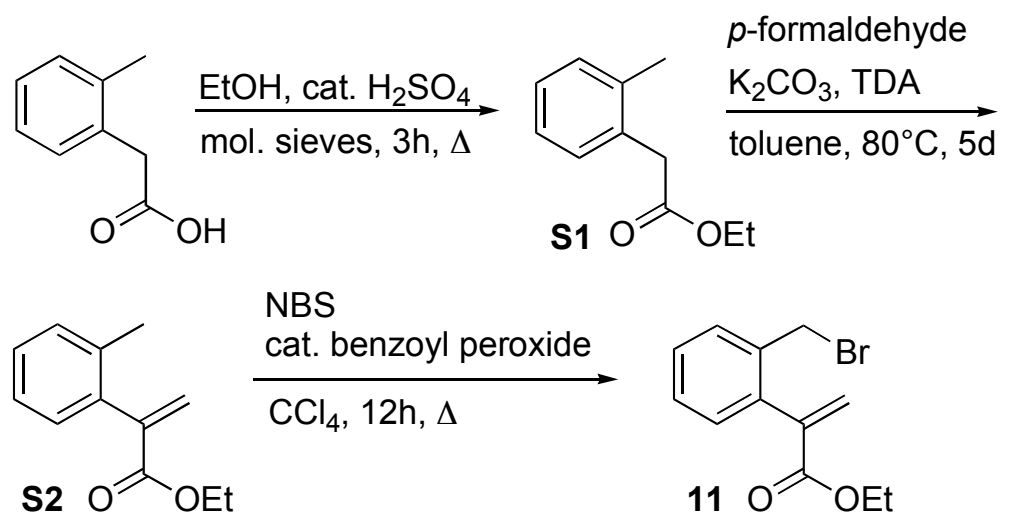<smiles>C=C(C(=O)OCC)c1ccccc1CBr</smiles>

Scheme A. Synthesis of ethyl 2-[2-(bromomethyl)phenyl]acrylate 11.

(S1) Ethyl (2-methylphenyl)acetate: To a solution of $o$-tolylacetic acid (60 mmol, $9 \mathrm{~g}$ ) in ethanol $(30 \mathrm{ml})$ is added conc. $\mathrm{H}_{2} \mathrm{SO}_{4}(2 \mathrm{ml})$ and molecular sieves $4 \AA(5 \mathrm{~g})$. The reaction mixture was refluxed for 3 hours and poured into $\mathrm{NH}_{4} \mathrm{Cl}(\mathrm{aq})$, diluted with ethylacetate $(30 \mathrm{ml})$ and the organic layer was separated. The aqueous layer was extracted two times with EtOAc $(20 \mathrm{ml})$ and the combined organic layers were dried over $\mathrm{MgSO}_{4}$, filtered and concentrated in vacuo. The crude product $\mathbf{S 1} 9.85 \mathrm{~g}(92 \%)$ is a colorless liquid. Bp.: $110^{\circ} \mathrm{C} / 15 \mathrm{~mm} ;{ }^{1} \mathrm{H}-\mathrm{NMR}(400 \mathrm{MHz}) \delta=7.16(\mathrm{~m}, 4 \mathrm{H}), 4.13(\mathrm{q}, J=$ 
$6.0 \mathrm{~Hz}, 2 \mathrm{H}), 3.61(\mathrm{~s}, 3 \mathrm{H}), 2.30(\mathrm{~s}, 3 \mathrm{H}), 1.23(\mathrm{t}, J=7.2 \mathrm{~Hz}, 3 \mathrm{H}) ;{ }^{13} \mathrm{C}-\mathrm{NMR}(100 \mathrm{MHz}) \delta=$ $171.4,136.7,132.8,130.2,130.0,127.2,126.0,60.7,39.2,19.5,14.1$; Ms (EI) for $\mathrm{C}_{10} \mathrm{H}_{12} \mathrm{O}_{2}: \mathrm{m} / \mathrm{z}=178.1\left(\mathrm{M}^{+}\right)$; HRMS calcd for $\mathrm{C}_{11} \mathrm{H}_{14} \mathrm{O}_{2} 178.0993$ found 178.0995 .

(S2) Ethyl 2-(2methylphenyl)acrylate ${ }^{1}$ : To a solution of $\mathbf{S 1}(40 \mathrm{mmol}, 9.5 \mathrm{~g})$, paraformaldehyde ( $800 \mathrm{mmol}, 24 \mathrm{~g}$ ), TDA-1 (tris[2-(2-methoxyethoxy)ethyl]amine) (4 mmol, $1.29 \mathrm{~g})$ and $\mathrm{K}_{2} \mathrm{CO}_{3}(200 \mathrm{mmol}, 27.6 \mathrm{~g})$ in $300 \mathrm{ml}$ dry toluene was stirred in a pressure vessel $(350 \mathrm{ml})$ at $85^{\circ} \mathrm{C}$. Full conversion, indicated by TLC, was achieved after five days. The cooled solution was filtered, washed thoroughly with water, dried over $\mathrm{MgSO}_{4}$, filtered and concentrated in vacuo. Purification by column chromatography $\left(\mathrm{SiO}_{2}\right.$ ethylacetate /hexane, $\left.1: 25, \mathrm{R}_{\mathrm{f}}=0.27\right)$ gave $5.8 \mathrm{~g}(76 \%)$ of $\mathbf{S 2}$ as a colorless oil. ${ }^{1} \mathrm{H}$ NMR (400 MHz) $\delta=7.25-7.15(\mathrm{~m}, 4 \mathrm{H}), 6.51(\mathrm{~d}, J=1.6 \mathrm{~Hz}, 1 \mathrm{H}), 5.70(\mathrm{~d}, J=1.6 \mathrm{~Hz}$, $1 \mathrm{H}), 4.25$ (q, $J=7.2 \mathrm{~Hz}, 2 \mathrm{H}), 2.24$ (s, 3H), 1.28 (t, $J=7.2 \mathrm{~Hz}, 3 \mathrm{H}) ;{ }^{13} \mathrm{C}-\mathrm{NMR}(100 \mathrm{MHz})$ $\delta=166.5,142.1,137.3,136.0,129.7,129.4,128.1,128.0,125.5,60.9,19.7,14.1$; Ms (EI) for $\mathrm{C}_{12} \mathrm{H}_{14} \mathrm{O}_{2}: \mathrm{m} / \mathrm{z}=190.2\left(\mathrm{M}^{+}\right)$; HRMS calcd for $\mathrm{C}_{12} \mathrm{H}_{14} \mathrm{O}_{2} 190.0993$, found 190.988 .

(11) Ethyl 2-[2-(bromomethyl)phenyl]acrylate: To a solution of $\mathbf{S 2}(30 \mathrm{mmol}, 5.7 \mathrm{~g})$ in $\mathrm{CCl}_{4}(30 \mathrm{ml})$ was added NBS (33 mmol, $\left.5.9 \mathrm{~g}\right)$ and benzoyl peroxide $(2 \mathrm{mmol}, 48 \mathrm{mg})$ and the reaction mixture was refluxed for $12 \mathrm{~h}$. The cooled solution was poured into water, separated and the water phase was extracted again with diethyl ether. The combined organic layers were dried over $\mathrm{MgSO}_{4}$, filtered and concentrated in vacuo. Purification by column chromatography $\left(\mathrm{SiO}_{2}\right.$ ethylacetate/hexane, 1:8, $\left.\mathrm{R}_{\mathrm{f}}=0.38\right)$ gave $7.6 \mathrm{~g}(88 \%)$ of 4 as a colorless oil. ${ }^{1} \mathrm{H}-\mathrm{NMR}(400 \mathrm{MHz}) \delta=7.43-7.13(\mathrm{~m}, 4 \mathrm{H}), 6.63(\mathrm{~s}$, $1 \mathrm{H}), 5.88(\mathrm{~s}, 1 \mathrm{H}), 4.42(\mathrm{~s}, 2 \mathrm{H}), 4.24(\mathrm{q}, J=6.8 \mathrm{~Hz}, 2 \mathrm{H}), 1.26(\mathrm{t}, J=6.8 \mathrm{~Hz}, 3 \mathrm{H}) ;{ }^{13} \mathrm{C}-$ NMR $(100 \mathrm{MHz}) \delta=166.2,139.6,137.0,135.6,130.4,130.2,129.8,128.6,128.3,61.3$, 31.6, 14.1; Ms (EI) for $\mathrm{C}_{12} \mathrm{H}_{13} \mathrm{BrO}_{2}: \mathrm{m} / \mathrm{z}=189.2\left(\mathrm{M}^{+}-\mathrm{HBr}\right)$; HRMS not possible because of instability.

(1) 2,2,2-Trifluoro-N-(2-iodo-phenyl)-acetamide: To a solution of 2-iodoaniline (4.38 g, $20 \mathrm{mmol})$ and $\mathrm{Et}_{3} \mathrm{~N}(4.2 \mathrm{ml}, 30 \mathrm{mmol})$ in $\mathrm{CH}_{2} \mathrm{Cl}_{2}(15 \mathrm{ml})$ was added slowly trifluoroacetic anhydride $(3.4 \mathrm{ml}, 24 \mathrm{mmol})$ at $0^{\circ} \mathrm{C}$. After stirring the reaction mixture for $1 \mathrm{~h}$ at the same temperature it was poured into $\mathrm{NH}_{4} \mathrm{Cl}$ (aq), diluted with ethylacetate (30 $\mathrm{ml}$ ) and the organic layer was separated. The aqueous layer was extracted two times with ethylacetate $(20 \mathrm{ml})$ and the combined organic layers were dried over $\mathrm{MgSO}_{4}$, filtered and concentrated in vacuo. Purification by crystallization from $\mathrm{H}_{2} \mathrm{O}$ gave $5.17 \mathrm{~g}(82 \%)$ of 1 as brownish solid $\left(\mathrm{mp} 102-103^{\circ} \mathrm{C}\right) .{ }^{1} \mathrm{H}-\mathrm{NMR}(400 \mathrm{MHz}) \delta=8.28(\mathrm{~s}$, broad, $1 \mathrm{H}(\mathrm{NH})$ ), $8.21(\mathrm{~d}, J=7.6 \mathrm{~Hz}, 1 \mathrm{H}), 7.84(\mathrm{~d}, J=7.6 \mathrm{~Hz}, 1 \mathrm{H}), 7.42(\mathrm{dd}, J=7.6 \mathrm{~Hz}, J=7.6 \mathrm{~Hz}, 1 \mathrm{H})$, $6.98(\mathrm{dd}, J=7.6 \mathrm{~Hz}, J=7.6 \mathrm{~Hz}, 1 \mathrm{H}) ;{ }^{13} \mathrm{C}-\mathrm{NMR}(100 \mathrm{MHz}) \delta=154.7\left(\mathrm{q},{ }^{2} J(C, F)=37\right.$ $\mathrm{Hz}), 139.1,135.6,129.5,127.8,122.2,115.5\left(\mathrm{q},{ }^{2} J(C, F)=289 \mathrm{~Hz}\right), 90.3 \mathrm{Ms}(\mathrm{EI})$ for $\mathrm{C}_{8} \mathrm{H}_{5} \mathrm{~F}_{3} \mathrm{INO}: \mathrm{m} / \mathrm{z}=315.1\left(\mathrm{M}^{+}\right)$; HRMS calcd for $\mathrm{C}_{8} \mathrm{H}_{5} \mathrm{~F}_{3} \mathrm{INO} 314.9368$, found 314.9370 .

(2) 2,2,2-Trifluoro-N-(2-bromo-phenyl)acetamide: To a solution of 2-bromoaniline (5 $\mathrm{mmol}, 860 \mathrm{mg})$ and $\mathrm{Et}_{3} \mathrm{~N}(7,5 \mathrm{mmol}, 1.04 \mathrm{ml})$ in $\mathrm{CH}_{2} \mathrm{Cl}_{2}(10 \mathrm{ml})$ was added slowly

${ }^{1}$ Carvotto, G.; Goivenzana, G.; Pilati, T.; Sisti, M.; Palmisano, G. J. Org. Chem. 2001, 66, 8447. 
trifluoroacetic anhydride $(5.5 \mathrm{mmol}, 776 \mu \mathrm{l})$ at $0^{\circ} \mathrm{C}$. After stirring the reaction mixture for $1 \mathrm{~h}$ at the same temperature it was poured into $\mathrm{NH}_{4} \mathrm{Cl}$ (aq), diluted with ethylacetate $(30 \mathrm{ml})$ and the organic layer was separated. The aqueous layer was extracted two times with ethylacetate $(20 \mathrm{ml})$ and the combined organic layers were dried over $\mathrm{MgSO}_{4}$, filtered and concentrated in vacuo to give $1.27 \mathrm{~g} \mathrm{(88 \% )}$ of crude 2 as a brownish solid $\left(\mathrm{mp} 57-59^{\circ} \mathrm{C}\right) .{ }^{1} \mathrm{H}-\mathrm{NMR}(400 \mathrm{MHz}) \delta=8.45(\mathrm{~s}$, broad, $1 \mathrm{H}(\mathrm{NH})), 8.24(\mathrm{~d}, J=7.8 \mathrm{~Hz}$, $1 \mathrm{H}), 7.56(\mathrm{~d}, J=7.8 \mathrm{~Hz}, 1 \mathrm{H}), 7.34(\mathrm{t}, J=7.8 \mathrm{~Hz}, 1 \mathrm{H}), 7.09(\mathrm{t}, J=7.8 \mathrm{~Hz}, 1 \mathrm{H}) ;{ }^{13} \mathrm{C}-\mathrm{NMR}$ $(100 \mathrm{MHz}) \delta=154.6\left(\mathrm{q},{ }^{2} J(C, F)=37 \mathrm{~Hz}\right), 133.1,132.6,128.7,128.6,127.2,122.1$, 119.9, 117.0, $115.6\left(\mathrm{q},{ }^{2} J(C, F)=289 \mathrm{~Hz}\right), 114.2$; Ms (EI) for $\mathrm{C}_{8} \mathrm{H}_{5} \mathrm{~F}_{3} \mathrm{INO}: \mathrm{m} / \mathrm{z}=315.1$ $\left(\mathrm{M}^{+}\right)$; HRMS calcd for $\mathrm{C}_{8} \mathrm{H}_{5} \mathrm{~F}_{3} \mathrm{INO} 314.9368$, found 314.9370 .

(5) Ethyl 2-(2-\{[(2-iodo-phenyl)-(2,2,2-trifluoro-acetyl)-amino]-methyl\}-phenyl)acrylate: To a solution of $\mathbf{1}(12 \mathrm{mmol}, 3.78 \mathrm{~g})$ and $\mathbf{1 1}(10.5 \mathrm{mmol}, 2.82 \mathrm{~g})$ in acetone $(100 \mathrm{ml})$ was added $\mathrm{K}_{2} \mathrm{CO}_{3}(55 \mathrm{mmol}, 7.6 \mathrm{~g})$ and the reaction mixture was refluxed for 18 h. The cooled solution was filtered and concentrated in vacuo. Purification by column chromatography $\left(\mathrm{SiO}_{2}\right.$ ethylacetate/hexane, $\left.1: 8, \mathrm{R}_{\mathrm{f}}=0.32\right)$ gave $4.28 \mathrm{~g}(71 \%)$ of 5 as a colorless oil. ${ }^{1} \mathrm{H}-\mathrm{NMR}(400 \mathrm{MHz}) \delta=7.88(\mathrm{~d}, J=8.0 \mathrm{~Hz}, 1 \mathrm{H}), 7.28-7.00(\mathrm{~m}, 6 \mathrm{H}), 6.62$ $(\mathrm{d}, J=7.6 \mathrm{~Hz}, 1 \mathrm{H}), 6.32(\mathrm{~d}, J=1.6 \mathrm{~Hz}, 1 \mathrm{H}), 5.75(\mathrm{~d}, J=14.4 \mathrm{~Hz}, 1 \mathrm{H}), 5.16(\mathrm{~d}, J=1.4$ $\mathrm{Hz}, 1 \mathrm{H}), 4.18(\mathrm{~d}, J=14.4 \mathrm{~Hz}, 1 \mathrm{H}), 4.14(\mathrm{q}, J=7.6 \mathrm{~Hz}, 2 \mathrm{H}), 1.27(\mathrm{t}, J=7.2 \mathrm{~Hz}, 3 \mathrm{H}) ;{ }^{13} \mathrm{C}-$ NMR $(100 \mathrm{MHz}) \delta=165.7,156.4\left(\mathrm{q},{ }^{2} J(C, F)=37 \mathrm{~Hz}\right), 139.9,139.7,139.5,137.8$, $133.0,131.3,130.4,130.0,129.9,129.2,128.4,128.3,128.0,115.5\left(\mathrm{q},{ }^{2} J(C, F)=287\right.$ $\mathrm{Hz})$, 99.4, 61.0, 49.6, 14.0. Ms (CI) for $\mathrm{C}_{20} \mathrm{H}_{17} \mathrm{~F}_{3} \mathrm{INO}_{3}: \mathrm{m} / \mathrm{z}=503.3\left(\mathrm{M}^{+}\right)$; HRMS calcd for $\mathrm{C}_{20} \mathrm{H}_{17} \mathrm{~F}_{3} \mathrm{INO}_{3}$ 503.0205, found 503.1095.

(6) Ethyl 2-(2-\{[(2-bromo-phenyl)-(2,2,2-trifluoro-acetyl)-amino]-methyl\}-phenyl)acrylate: To a solution of $2(2.5 \mathrm{mmol}, 787 \mathrm{mg})$ and $11(3 \mathrm{mmol}, 804 \mathrm{mg})$ in acetone (30 $\mathrm{ml})$ was added $\mathrm{K}_{2} \mathrm{CO}_{3}(15 \mathrm{mmol}, 2.07 \mathrm{~g})$ and the reaction mixture was refluxed for $18 \mathrm{~h}$. The cooled solution was filtered and concentrated in vacuo. Purification by column chromatography $\left(\mathrm{SiO}_{2}\right.$ ethylacetate/hexane, $\left.1: 10, \mathrm{R}_{\mathrm{f}}=0.33\right)$ gave $694 \mathrm{mg}(77 \%)$ of $\mathbf{6}$ as a colorless oil. ${ }^{1} \mathrm{H}-\mathrm{NMR}(400 \mathrm{MHz}) \delta=7.60(\mathrm{~d}, J=7.8 \mathrm{~Hz}, 1 \mathrm{H}), 7.25-7.04(\mathrm{~m}, 6 \mathrm{H}), 6.66$ (d, $J=7.8 \mathrm{~Hz}, 1 \mathrm{H}), 6.32(\mathrm{~d}, J=1.4 \mathrm{~Hz}, 1 \mathrm{H}), 5.73(\mathrm{~d}, J=14.5 \mathrm{~Hz}, 1 \mathrm{H}), 5.19$ (d, $J=1.4$ $\mathrm{Hz}, 1 \mathrm{H}), 4.15(\mathrm{q}, J=6.9 \mathrm{~Hz}, 2 \mathrm{H}), 4.13(\mathrm{~d}, J=14.4 \mathrm{~Hz}, 1 \mathrm{H}), 1.24(\mathrm{t}, J=6.9 \mathrm{~Hz}, 3 \mathrm{H}) ;{ }^{13} \mathrm{C}-$ $\operatorname{NMR}(100 \mathrm{MHz}) \delta=165.8,156.6\left(\mathrm{q},{ }^{2} J(C, F)=36 \mathrm{~Hz}\right), 140.0,137.8,136.3,133.2$, $133.1,131.8,130.5,130.1,130.0,129.2,128.3,128.0,127.5,116.1\left(\mathrm{q},{ }^{2} J(C, F)=289\right.$ $\mathrm{Hz}), 61.1,49.4$, 14.0. Ms (CI) for $\mathrm{C}_{20} \mathrm{H}_{17} \mathrm{BrF}_{3} \mathrm{NO}_{3}: \mathrm{m} / \mathrm{z}=456.1\left(\mathrm{M}+\mathrm{H}^{+}\right)$; HRMS calcd for $\mathrm{C}_{20} \mathrm{H}_{17} \mathrm{BrF}_{3} \mathrm{NO}_{3}\left(+\mathrm{H}^{+}\right)$456.0417, found 456.0405 .

(7)thyl 2-\{2-[(2-iodophenoxy)methyl]phenyl\}acrylate: To a solution of 2Iodophenol $(2 \mathrm{mmol}, 538 \mathrm{mg})$ and $\mathbf{1 1}(2 \mathrm{mmol}, 440 \mathrm{mg})$ in acetone $(10 \mathrm{ml})$ was added $\mathrm{K}_{2} \mathrm{CO}_{3}(5 \mathrm{mmol}, 690 \mathrm{mg})$ and the reaction mixture was refluxed for $6 \mathrm{~h}$. The cooled solution was filtered and concentrated in vacuo. Purification by column chromatography $\left(\mathrm{SiO}_{2}\right.$ ethylacetate/hexane, 1:12, $\left.\mathrm{R}_{\mathrm{f}}=0.20\right)$ gave $725 \mathrm{mg}(84 \%)$ of 7 as a colorless oil. ${ }^{1} \mathrm{H}$ NMR $(400 \mathrm{MHz}) \delta=7.76(\mathrm{~d}, J=7.8 \mathrm{~Hz}, 1 \mathrm{H}), 7.65(\mathrm{~d}, J=7.6 \mathrm{~Hz}, 1 \mathrm{H}), 7.38(\mathrm{t}, J=6.7$ $\mathrm{Hz}, 1 \mathrm{H}), 7.31(\mathrm{t}, J=6.5 \mathrm{~Hz}, 1 \mathrm{H}), 7.20(\mathrm{t}, J=6.9 \mathrm{~Hz}, 1 \mathrm{H}), 6.75(\mathrm{~d}, J=8.1 \mathrm{~Hz}, 1 \mathrm{H}), 6.69$ $(\mathrm{t}, J=7.8 \mathrm{~Hz}, 1 \mathrm{H}), 6.56(\mathrm{~d}, J=1.9 \mathrm{~Hz}, 1 \mathrm{H}), 5.83(\mathrm{~d}, J=1.9 \mathrm{~Hz}, 1 \mathrm{H}), 5.03(\mathrm{~s}, 2 \mathrm{H}), 4.21$ $(\mathrm{q}, J=6.9 \mathrm{~Hz}, 2 \mathrm{H}), 1.25(\mathrm{t}, J=6.8 \mathrm{~Hz}, 3 \mathrm{H}) ;{ }^{13} \mathrm{C}-\mathrm{NMR}(100 \mathrm{MHz}) \delta=166.2,157.0$, 
$140.3,139.5,135.9,134.5,129.8,129.6,129.3,128.4,127.7,127.6,122.7,112.5,86.5$, 68.8, 61.3, 14.1.HRMS calcd for $\mathrm{C}_{20} \mathrm{H}_{17} \mathrm{IO}_{3}\left(+\mathrm{Na}^{+}\right) 431.0120$, found 431.0110 .

(8) Ethyl 2-\{2-[(2bromophenoxy)methyl]phenyl\}acrylate: To a solution of 11 (2.5 $\mathrm{mmol}, 432 \mathrm{mg}$ ) and 2-bromophenol (3 mmol, $804 \mathrm{mg})$ in acetone $(20 \mathrm{ml})$ was added $\mathrm{K}_{2} \mathrm{CO}_{3}(15 \mathrm{mmol}, 2.07 \mathrm{~g})$ and the reaction mixture was reflux for $18 \mathrm{~h}$. The cooled solution was filtered and concentrated in vacuo. Purification by column chromatography $\left(\mathrm{SiO}_{2}\right.$ ethylacetate/hexane, $\left.3: 40, \mathrm{R}_{\mathrm{f}}=0.35\right)$ gave $784 \mathrm{mg}(69 \%)$ of $\mathbf{8}$ as a colorless oil. ${ }^{1} \mathrm{H}-$ NMR $(400 \mathrm{MHz}) \delta=7.63(\mathrm{~d}, J=7.8 \mathrm{~Hz}, 1 \mathrm{H}), 7.53(\mathrm{~d}, J=7.6 \mathrm{~Hz}, 1 \mathrm{H}), 7.39(\mathrm{t}, J=6.7$ $\mathrm{Hz}, 1 \mathrm{H}), 7.34(\mathrm{t}, J=6.5 \mathrm{~Hz}, 1 \mathrm{H}), 7.20(\mathrm{~m}, 2 \mathrm{H}), 6.82(\mathrm{~m}, 2 \mathrm{H}), 6.57(\mathrm{~d}, J=1.9 \mathrm{~Hz}, 1 \mathrm{H})$, $5.83(\mathrm{~d}, J=1.9 \mathrm{~Hz}, 1 \mathrm{H}), 5.05(\mathrm{~s}, 2 \mathrm{H}), 4.23(\mathrm{q}, J=6.9 \mathrm{~Hz}, 2 \mathrm{H}), 1.26(\mathrm{t}, J=6.8 \mathrm{~Hz}, 3 \mathrm{H})$; ${ }^{13} \mathrm{C}-\mathrm{NMR}(100 \mathrm{MHz}) \delta=166.1,157.7,140.3,136.0,134.4,133.3,129.8,129.4,128.4$, 128.3, 127.7, 127.6, 122.0, 113.5, 112.2, 68.7, 61.2, 14.1. HRMS calcd for $\mathrm{C}_{18} \mathrm{H}_{17} \mathrm{BrO}_{3}$ $\left(+\mathrm{Na}^{+}\right) 383.0253$, found 383.0269.

(9) Ethyl 5-(trifluoroacetyl)-5,6-dihydrodibenzo[b,f]azocine-11-carboxylate: Under argon atmosphere: To a solution of $5(0.33 \mathrm{mmol}, 166 \mathrm{mg})$ in dimethylacetamide $(4 \mathrm{ml})$ was added $\mathrm{Et}_{4} \mathrm{NCl}(0.3 \mathrm{mmol}, 50 \mathrm{mg}), \mathrm{N}$-methylcyclohexylamine $(0.45 \mathrm{mmol}, 96 \mu \mathrm{l})$ and $\mathrm{Pd}(\mathrm{OAc})_{2}(0.0165 \mathrm{mmol}, 3.7 \mathrm{mg})$. After stirring reaction mixture for $4 \mathrm{~h}$ at $95^{\circ} \mathrm{C}$ the reaction mixture was poured into water $(10 \mathrm{ml})$ and the extracted with ethylacetate $(2 \times 20$ $\mathrm{ml})$. The combined organic layers were dried over $\mathrm{MgSO}_{4}$, filtered and concentrated in vacuo. Purified by column chromatography $\left(\mathrm{SiO}_{2}\right.$ ethylacetate/hexane, $\left.1: 8, \mathrm{R}_{\mathrm{f}}=0.29\right)$ to give $89 \mathrm{mg}(72 \%)$ of 8 as colorless oil. ${ }^{1} \mathrm{H}-\mathrm{NMR}(400 \mathrm{MHz}) \delta=7.91(\mathrm{~s}, 1 \mathrm{H}), 7.23-7.01$ $(\mathrm{m}, 8 \mathrm{H}), 5.07$ (d, $J=14.3 \mathrm{~Hz}, 1 \mathrm{H}), 4.55(\mathrm{~d}, J=14.3 \mathrm{~Hz}, 1 \mathrm{H}), 4.29(\mathrm{q}, J=6.9 \mathrm{~Hz}, 2 \mathrm{H})$, $1.31(\mathrm{t}, J=7.2 \mathrm{~Hz}, 3 \mathrm{H}) ;{ }^{13} \mathrm{C}-\mathrm{NMR}(100 \mathrm{MHz}) \delta=165.4,156.2\left(\mathrm{q},{ }^{2} J(C, F)=36 \mathrm{~Hz}\right)$, 140.2 , 137.2, 137.1, 136.4, 135.1, 131.3, 130.5, 129.5, 129.4, 129.1, 128.9, 128.4, 128.2, $127.9,127.0,115.8\left(\mathrm{q},{ }^{2} J(C, F)=287 \mathrm{~Hz}\right), 61.6,55.2,14.1$; HRMS calcd for $\mathrm{C}_{20} \mathrm{H}_{17} \mathrm{~F}_{3} \mathrm{INO}_{3} 375.1082$, found 375.1079 .

(10) Ethyl 6H-dibenzo[b,f]oxocine-11-carboxylate: Under argon atmosphere: To a solution of $7(0.33 \mathrm{mmol}, 134 \mathrm{mg})$ in dimethylacetamide $(4 \mathrm{ml})$ was added $\mathrm{Et}_{4} \mathrm{NCl}(0.3$ mmol, $50 \mathrm{mg})$, N-methylcyclohexylamine $(0.45 \mathrm{mmol}, 96 \mu \mathrm{l})$ and $\operatorname{Pd}(\mathrm{OAc})_{2}(0.0165$ mmol, $3.7 \mathrm{mg}$ ). After stirring reaction mixture for $4 \mathrm{~h}$ at $95^{\circ} \mathrm{C}$ the reaction mixture was poured into water $(10 \mathrm{ml})$ and the extracted with ethylacetate $(2 \times 20 \mathrm{ml})$. The combined organic layers were dried over $\mathrm{MgSO}_{4}$, filtered and concentrated in vacuo. Purified by column chromatography $\left(\mathrm{SiO}_{2}\right.$ ethylacetate/hexane, $\left.1: 12, \mathrm{R}_{\mathrm{f}}=0.27\right)$ to give $76 \mathrm{mg}(82 \%)$ of 10 as colorless oil. ${ }^{1} \mathrm{H}-\mathrm{NMR}(400 \mathrm{MHz}) \delta=7.94(\mathrm{~s}, 1 \mathrm{H}), 7.48(\mathrm{~m}, 1 \mathrm{H}), 7.31(\mathrm{~m}, 3 \mathrm{H})$, $7.01(\mathrm{~m}, 2 \mathrm{H}), 6.82(\mathrm{~m}, 2 \mathrm{H}), 5.33(\mathrm{~s}, 2 \mathrm{H}), 4.30(\mathrm{q}, J=7.2 \mathrm{~Hz}, 2 \mathrm{H}), 1.32(\mathrm{t}, J=7.8 \mathrm{~Hz}$, $3 \mathrm{H}) ;{ }^{13} \mathrm{C}-\mathrm{NMR}(100 \mathrm{MHz}) \delta=166.7,154.8,141.7,136.6,134.8,132.1,131.1,130.4$, $129.9,129.8,128.4,128.2,122.1,120.9,120.4,70.2,61.3,14.2$; Ms (CI) for $\mathrm{C}_{18} \mathrm{H}_{16} \mathrm{O}_{3}$ : $\mathrm{m} / \mathrm{z}=281.1\left(\mathrm{M}+\mathrm{H}^{+}\right)$; HRMS calcd for $\mathrm{C}_{18} \mathrm{H}_{16} \mathrm{O}_{3} 280.1099$, found 280.1099. 


\section{Materials described in Scheme 2.}

(12) 2-Iodo-benzenesulfonyl chloride ${ }^{2}:{ }^{1} \mathrm{H}-\mathrm{NMR}$ (400 $\left.\mathrm{MHz}\right) \mathrm{CDCl}_{3} \delta=8.21$ (dd, $J=$ $7.6 \mathrm{~Hz}, J=7.6 \mathrm{~Hz}, 2 \mathrm{H}), 7.59(\mathrm{t}, J=7.6 \mathrm{~Hz}, 1 \mathrm{H}), 7.35$ (t, $J=7.6 \mathrm{~Hz}, 1 \mathrm{H}),{ }^{13} \mathrm{C}-\mathrm{NMR}(100$ MHz) $\delta=164.1,146.7,135.5,130.3,128.8,92.0$; HRMS calcd for $\mathrm{C}_{6} \mathrm{H}_{4} \mathrm{ClIO}_{2} \mathrm{~S}[(\mathrm{M}-$ $\mathrm{HCl})]$ 266.8971, found 266.8975.

(14) Ethyl 2-(2-\{[(@dophenyl)sulfonyl]methyl\}phenyl)acrylate: $\mathrm{Na}_{2} \mathrm{HPO}_{4} \quad$ (4 mmol, $567 \mathrm{mg}), \mathrm{Na}_{2} \mathrm{SO}_{3}(8 \mathrm{mmol}, 1 \mathrm{~g})$ and 12 (4 mmol, $\left.1.21 \mathrm{~g}\right)$ were dissolved water (4 $\mathrm{ml})$ and stirred for $16 \mathrm{~h}$ at $60^{\circ} \mathrm{C} .11(2 \mathrm{mmol}, 538 \mathrm{mg})$ in acetone $(1 \mathrm{ml})$ was added stirring was continued for $16 \mathrm{~h}$. The reaction mixture was poured into water $(10 \mathrm{ml})$ and the extracted with ethylacetate $(2 \times 20 \mathrm{ml})$. The combined organic layers were dried over $\mathrm{MgSO}_{4}$, filtered and concentrated in vacuo. Purified by column chromatography $\left(\mathrm{SiO}_{2}\right.$ ethylacetate/hexane, 1:2, $\left.\mathrm{R}_{\mathrm{f}}=0.41\right)$ to give $14420 \mathrm{mg}(46 \%)$ as a colorless oil. ${ }^{1} \mathrm{H}-\mathrm{NMR}$ $(400 \mathrm{MHz}) \delta=8.07(\mathrm{dd}, J=7.6 \mathrm{~Hz}, J=1.2 \mathrm{~Hz}, 1 \mathrm{H}), 7.80(\mathrm{dd}, J=7.6 \mathrm{~Hz}, J=1.6 \mathrm{~Hz}$, $1 \mathrm{H}), 7.39-7.20(\mathrm{~m}, 7 \mathrm{H}), 7.10(\mathrm{dd}, J=7.6 \mathrm{~Hz}, J=1.6 \mathrm{~Hz}, 1 \mathrm{H}), 6.51(\mathrm{~d}, J=1.6 \mathrm{~Hz}, 1 \mathrm{H})$, $5.52(\mathrm{~d}, J=1.6 \mathrm{~Hz}, 1 \mathrm{H}), 4.62(\mathrm{~s}, 2 \mathrm{H}), 4.20(\mathrm{q}, J=7.2 \mathrm{~Hz}, 2 \mathrm{H}), 1.27(\mathrm{t}, J=7.2 \mathrm{~Hz}, 3 \mathrm{H})$; ${ }^{13} \mathrm{C}-\mathrm{NMR}(100 \mathrm{MHz}) \delta=165.7,142.5,140.7,139.3,138.6,134.3,132.1,131.8,131.1$, $130.4,128.5,128.4,128.0,125.8,93.4,61.1,56.5,14.1$; HRMS calcd for $\mathrm{C}_{18} \mathrm{H}_{17} \mathrm{IO}_{4} \mathrm{~S}$ $(\mathrm{M}+\mathrm{Na})^{+} 456.9965$, found 456.9960 .

(15) Ethyl 2-(2-\{[(Bromophenyl)sulfonyl]methyl $\}$ phenyl)acrylate: $\mathrm{Na}_{2} \mathrm{HPO}_{4}$ (2 mmol, $284 \mathrm{mg}$ ), $\mathrm{Na}_{2} \mathrm{SO}_{3}$ (4 mmol, $504 \mathrm{mg}$ ) and 13 (2 mmol, $511 \mathrm{mg}$ ) were dissolved water $(2 \mathrm{ml})$ and stirred for $16 \mathrm{~h}$ at $60^{\circ} \mathrm{C} .11(2 \mathrm{mmol}, 538 \mathrm{mg})$ in acetone $(1 \mathrm{ml})$ was added stirring was continued for $16 \mathrm{~h}$. The reaction mixture was poured into water $(10 \mathrm{ml})$ and the extracted with ethylacetate $(2 \times 20 \mathrm{ml})$. The combined organic layers were dried over $\mathrm{MgSO}_{4}$, filtered and concentrated in vacuo. Purified by column chromatography $\left(\mathrm{SiO}_{2}\right.$ ethylacetate/hexane, 1:2, $\left.\mathrm{R}_{\mathrm{f}}=0.42\right)$ to give $\mathbf{A 3 0} 290 \mathrm{mg}(35 \%)$ as a colorless oil. ${ }^{1} \mathrm{H}-\mathrm{NMR}(400 \mathrm{MHz}) \delta=7.83(\mathrm{dd}, J=7.6 \mathrm{~Hz}, J=1.6 \mathrm{~Hz}, 1 \mathrm{H}), 7.74(\mathrm{dd}, J=7.6 \mathrm{~Hz}, J=$ $1.2 \mathrm{~Hz}, 1 \mathrm{H}), 7.45-7.35(\mathrm{~m}, 4 \mathrm{H}), 7.31-7.26(\mathrm{~m}, 3 \mathrm{H}), 7.12(\mathrm{dd}, J=7.6 \mathrm{~Hz}, J=1.6 \mathrm{~Hz}, 1 \mathrm{H})$, $6.51(\mathrm{~d}, J=1.6 \mathrm{~Hz}, 1 \mathrm{H}), 5.59(\mathrm{~d}, J=1.6 \mathrm{~Hz}, 1 \mathrm{H}), 4.66(\mathrm{~s}, 2 \mathrm{H}), 4.21(\mathrm{q}, J=7.2 \mathrm{~Hz}, 2 \mathrm{H})$, $1.28(\mathrm{t}, J=7.2 \mathrm{~Hz}, 3 \mathrm{H}) ;{ }^{13} \mathrm{C}-\mathrm{NMR}(100 \mathrm{MHz}) \delta=165.8,139.4,138.7,137.9,135.3$, $134.7,132.5,131.8,131.1,130.5,128.6,128.1,127.7,125.8,121.4,61.2,57.2,14.1$; HRMS calcd for $\mathrm{C}_{18} \mathrm{H}_{17} \mathrm{BrO}_{4} \mathrm{~S}(\mathrm{M}+\mathrm{Li})$ 415.1915, found 415.200.

(16) Ethyl 6H-dibenbof|thiocine-11-carboxylate 5,5-dioxide: Under argon atmosphere: To a solution of $14(0.26 \mathrm{mmol}, 119 \mathrm{mg})$ in dimethylacetamide $(3 \mathrm{ml})$ was added $\mathrm{Et}_{4} \mathrm{NCl}(0.3 \mathrm{mmol}, 50 \mathrm{mg})$, N-methylcyclo-hexylamine $(0.45 \mathrm{mmol}, 0.115 \mathrm{ml})$ and $\mathrm{Pd}(\mathrm{OAc})_{2}(0.013 \mathrm{mmol}, 2.9 \mathrm{mg})$. After stirring reaction mixture for $18 \mathrm{~h}$ at $100^{\circ} \mathrm{C}$ the reaction mixture was poured into water $(10 \mathrm{ml})$ and the extracted with ethylacetate $(2 \times 20$ $\mathrm{ml})$. The combined organic layers were dried over $\mathrm{MgSO}_{4}$, filtered and concentrated in vacuo. Purified by column chromatography $\left(\mathrm{SiO}_{2}\right.$ ethylacetate/hexane, $\left.1: 2 \mathrm{R}_{\mathrm{f}}=0.41\right)$ to

${ }^{2}$ Chau, M. M., Kice, J. L. J. Org. Chem. 1977, 42, 3265. 
give $59 \mathrm{mg}(69 \%)$ of 16 as a colorless oil. ${ }^{1} \mathrm{H}-\mathrm{NMR}(400 \mathrm{MHz}) \delta=8.23(\mathrm{~s}, 1 \mathrm{H}), 7.93(\mathrm{~d}$, $J=7.6 \mathrm{~Hz}, 1 \mathrm{H}), 7.56(\mathrm{~d}, J=7.6 \mathrm{~Hz}, 1 \mathrm{H}), 7.43-7.18(\mathrm{~m}, 5 \mathrm{H}), 7.07(\mathrm{~d}, J=7.6 \mathrm{~Hz}, 1 \mathrm{H})$, $4.74(\mathrm{~d}, J=13.6 \mathrm{~Hz}, 1 \mathrm{H}), 4.58(\mathrm{~d}, J=13.6 \mathrm{~Hz}, 1 \mathrm{H}), 4.31(\mathrm{q}, J=7.2 \mathrm{~Hz}, 2 \mathrm{H}), 1.34(\mathrm{t}, J=$ $7.2 \mathrm{~Hz}, 3 \mathrm{H}) ;{ }^{13} \mathrm{C}-\mathrm{NMR}(100 \mathrm{MHz}) \delta=165.3,140.9,138.3,136.7,136.1,133.7,132.4$, 129.8, 129.1, 129.0, 128.9, 128.7, 128.4, 127.1, 64.7, 61.9, 14.1; HRMS calcd for $\mathrm{C}_{18} \mathrm{H}_{16} \mathrm{O}_{4} \mathrm{~S}(\mathrm{M}+\mathrm{Li}) 335.0929$, found 335.0930.

\section{Materials described in Scheme 3.}
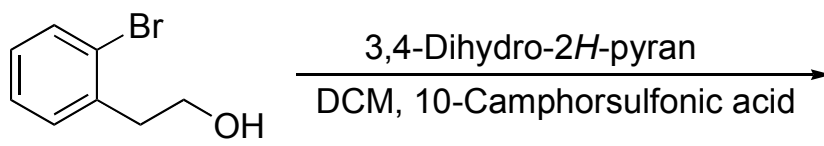

1. $n$-BuLi, THF ethyl pyruvate

2. $\mathrm{POCl}_{3}$, pyridine

3. $\mathrm{MeOH}, \mathrm{p}-\mathrm{TsOH}$

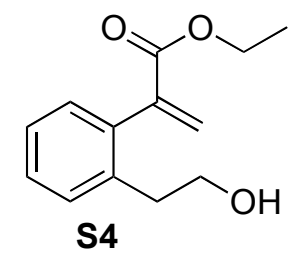

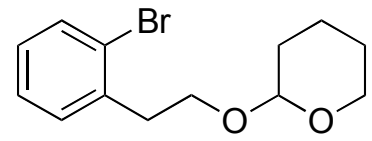

S3

Scheme B. Synthesis of S4.

(S3) 2-[2-(2Bromophenyl)ethoxy]tetrahydro-2H-pyran: To a stirred solution of 2bromophenethyl alcohol $(21 \mathrm{~g}, 105 \mathrm{mmol})$ and 10-camphorsulfonic acid (500 $\mathrm{mg}, 2$ $\mathrm{mmol})$ in DCM $(250 \mathrm{ml})$ was added 3,4-dihydro- $2 H$-pyran $(13.7 \mathrm{ml}, 150 \mathrm{mmol})$ in DMC $(20 \mathrm{ml})$ at $\mathrm{rt}$. After stirring over night the solvent was evaporated and the residue was purified by column chromatography $\left(\mathrm{SiO}_{2}\right.$ ethylacetate/hexane, 1:15, $\left.\mathrm{R}_{\mathrm{f}}=0.35\right)$ to give $28.22 \mathrm{~g} \mathrm{S3}(99 \%)$ as a colorless liquid. ${ }^{1} \mathrm{H}-\mathrm{NMR}(400 \mathrm{MHz}) \delta=7.50(\mathrm{~d}, J=7.7 \mathrm{~Hz}, 1 \mathrm{H})$, $7.28(\mathrm{~d}, J=7.7 \mathrm{~Hz}, 1 \mathrm{H}), 7.23(\mathrm{t}, J=7.7 \mathrm{~Hz}, 1 \mathrm{H}), 7.06(\mathrm{t}, J=7.7 \mathrm{~Hz}, 1 \mathrm{H}), 4.61(\mathrm{~m}, 1 \mathrm{H})$, $3.96(\mathrm{~m}, 1 \mathrm{H}), 3.76(\mathrm{~m}, 1 \mathrm{H}), 3.64(\mathrm{~m}, 1 \mathrm{H}), 3.46(\mathrm{~m}, 1 \mathrm{H}), 3.06(\mathrm{t}, J=7.3 \mathrm{~Hz}, 3 \mathrm{H}), 1.83-$ $1.46(\mathrm{~m}, 6 \mathrm{H}) ;{ }^{13} \mathrm{C}-\mathrm{NMR}(100 \mathrm{MHz}) \delta=132.6,131.4,128.2,127.8,127.2,124.6,98.5$, 66.3, 62.0, 36.4, 30.6, 25.4, 19.4; HRMS calcd for $\mathrm{C}_{13} \mathrm{H}_{17} \mathrm{BrO}_{2}\left(\mathrm{Na}^{+}\right) 310.0310$ found 310.0323 .

(S4) Ethyl 2-[2-(2-hydroxyethyl)phenyl]acrylate: To a solution of S3 (104 mmol, 28 g) in THF $(300 \mathrm{ml})$ was added $\mathrm{n}-\mathrm{BuLi}(44 \mathrm{ml}, 2.5 \mathrm{M}$ in hexanes) over a period of $1 \mathrm{~h}$ at $-78^{\circ} \mathrm{C}$ followed by the addition of ethyl pyruvate $(120 \mathrm{mmol}, 13.3 \mathrm{ml})$ over a period of 1 $\mathrm{h}$ at the same temperature. The reaction mixture was warmed up to $\mathrm{rt}$ and stirred for an additional $3 \mathrm{~h}$, poured into $\mathrm{NH}_{4} \mathrm{Cl}$ (aq) and extracted with ethyl acetate two times. The combined organic layers were washed with brine, dried over $\mathrm{MgSO}_{4}$, filtered and 
concentrated in vacuo. The crude product was dissolved in pyridine $(200 \mathrm{ml})$ and $\mathrm{POCl}_{3}$ $(150 \mathrm{mmol}, 22 \mathrm{ml})$ was added at $0^{\circ} \mathrm{C}$. The reaction mixture was warmed up to $50^{\circ} \mathrm{C}$ and stirred over night. After evaporation of the solvent the residue was dissolved in ethyl acetate and washed with $\mathrm{NH}_{4} \mathrm{Cl}(\mathrm{aq})$, brine, dried over $\mathrm{MgSO}_{4}$, filtered and concentrated in vacuo. The crude product was further dissolved in $\mathrm{MeOH}$ followed by the addition of $\mathrm{p}$-TsOH $(300 \mathrm{mg})$. The reaction mixture was stirred over night $\mathrm{rt}$ and the solvent was evaporated. Purification of the residue by column chromatography $\left(\mathrm{SiO}_{2}\right.$ ethyl acetate/hexane, 1:2, $\left.\mathrm{R}_{\mathrm{f}}=0.24\right)$ gave $5.74 \mathrm{~g}(25 \%$ over three steps $)$ of $\mathbf{S 4}$ as a colorless oil. ${ }^{1} \mathrm{H}-\mathrm{NMR}(400 \mathrm{MHz}) \delta=7.31-7.23(\mathrm{~m}, 2 \mathrm{H}), 7.22(\mathrm{dt}, J=7.8 \mathrm{~Hz}, J=1.8 \mathrm{~Hz}, 1 \mathrm{H}), 7.14$ $(\mathrm{dd}, J=7.8 \mathrm{~Hz}, J=1.5 \mathrm{~Hz}, 1 \mathrm{H}), 6.53(\mathrm{~d}, J=1.8 \mathrm{~Hz}, 1 \mathrm{H}), 5.72(\mathrm{~d}, J=1.5 \mathrm{~Hz}, 1 \mathrm{H}), 4.23$ $(\mathrm{q}, J=6.8 \mathrm{~Hz}, 2 \mathrm{H}), 3.78(\mathrm{t}, J=6.8 \mathrm{~Hz}, 2 \mathrm{H}), 2.77(\mathrm{t}, J=6.8 \mathrm{~Hz}, 2 \mathrm{H}), 1.27(\mathrm{t}, J=6.8 \mathrm{~Hz}$, $3 \mathrm{H}) ;{ }^{13} \mathrm{C}-\mathrm{NMR}$ (100 MHz) 166.7, 141.3, 137.2, 136.4, 129.7, 129.2, 128.7, 128.0, 126.0, 62.1, 61.0, 36.2, 13.9; HRMS calcd for $\mathrm{C}_{13} \mathrm{H}_{16} \mathrm{O}_{3} 221.1172$ found 221.1174.

(17) Ethyl 2-\{2-[2-(2-iodophenoxy)ethyl]phenyl\}acrylate: To a solution of S4 (1 mmol, $220 \mathrm{mg})$, 2-iodophenol (1 mmol, $219 \mathrm{mg})$ and $\mathrm{PPh}_{3}(1.3 \mathrm{mmol}, 342 \mathrm{mg})$ in THF $(10 \mathrm{ml})$ was added DIAD $(1.2 \mathrm{mmol}, 0.236 \mathrm{ml})$ at $0^{\circ} \mathrm{C}$. After stirring for $15 \mathrm{~min}$ the reaction mixture was warmed up to $\mathrm{rt}$ and stirred for an additional $3 \mathrm{~h}$. The solvent was evaporated and the residue was purified by column chromatography $\left(\mathrm{SiO}_{2}\right.$ ethyl acetate/hexane, 1:8, $\left.\mathrm{R}_{\mathrm{f}}=0.36\right)$ to give $333 \mathrm{mg}$ of $17(79 \%)$ as a colorless oil. ${ }^{1} \mathrm{H}-\mathrm{NMR}$ $(400 \mathrm{MHz}) \delta=7.74(\mathrm{~d}, J=7.7 \mathrm{~Hz}, 1 \mathrm{H}), 7.45(\mathrm{~d}, J=7.7 \mathrm{~Hz}, 1 \mathrm{H}), 7.31(\mathrm{t}, J=7.7 \mathrm{~Hz}, 1 \mathrm{H})$, $7.23(\mathrm{~m}, 2 \mathrm{H}), 7.15(\mathrm{~d}, J=7.7 \mathrm{~Hz}, 1 \mathrm{H}), 6.73(\mathrm{~d}, J=7.7 \mathrm{~Hz}, 1 \mathrm{H}), 6.67(\mathrm{t}, J=7.7 \mathrm{~Hz}, 1 \mathrm{H})$, $6.65(\mathrm{~d}, J=1.4 \mathrm{~Hz}, 1 \mathrm{H}), 5.76(\mathrm{~d}, J=1.4 \mathrm{~Hz}, 1 \mathrm{H}), 4.22(\mathrm{~d}, J=6.9 \mathrm{~Hz}, 2 \mathrm{H}), 4.12(\mathrm{t}, J=$ $6.9 \mathrm{~Hz}, 2 \mathrm{H}), 3.07(\mathrm{~d}, J=6.9 \mathrm{~Hz}, 2 \mathrm{H}), 1.26(\mathrm{t}, J=6.9 \mathrm{~Hz}, 3 \mathrm{H}) ;{ }^{13} \mathrm{C}-\mathrm{NMR}(100 \mathrm{MHz}) \delta=$ $166.5,157.3,141.4,139.4,137.2,135.8,130.1$, 129.9, 129.3, 129.1, 128.4, 128.2, 126.5 , 122.5, 112.1, 86.5, 74.3, 61.2, 32.9, 14.1; HRMS calcd for $\mathrm{C}_{19} \mathrm{H}_{19} \mathrm{IO}_{3} 422.0379$ found 422.0382 .

(18) 2-[1-(Ethoxycarbonyl)vinyl]benzyl 2-iodobenzoate: A mixture of 2-iodobenzoic acid (2 mmol, $496 \mathrm{mg}), \mathrm{CsF}$ (3 mmol, $453 \mathrm{mg}), \mathbf{A 6}(2 \mathrm{mmol}, 538 \mathrm{mg})$, and DMF (5 ml) was stirred at $\mathrm{rt}$ for $24 \mathrm{~h}$. the reaction mixture was combined with $\mathrm{NaHCO}_{3}$ (aq) and extracted two times with EtOAc. The combined organic layers were dried over $\mathrm{MgSO}_{4}$, filtered and concentrated in vacuo. Purification by column chromatography $\left(\mathrm{SiO}_{2}\right.$ ethylacetate/hexane, 1:14, $\left.\mathrm{R}_{\mathrm{f}}=0.13\right)$ gave $668 \mathrm{mg}(95 \%)$ of $\mathbf{A 1 5}$ as a colorless oil. ${ }^{1} \mathrm{H}$ NMR $(400 \mathrm{MHz}) \delta=7.99(\mathrm{~d}, J=7.6 \mathrm{~Hz}, 1 \mathrm{H}), 7.80(\mathrm{~d}, J=7.8 \mathrm{~Hz}, 1 \mathrm{H}), 7.53(\mathrm{~d}, J=6.6$ $\mathrm{Hz}, 1 \mathrm{H}), 7.40-7.36(\mathrm{~m}, 3 \mathrm{H}), 7.22(\mathrm{~d}, J=7.8 \mathrm{~Hz}, 1 \mathrm{H}), 7.15(\mathrm{t}, J=7.8 \mathrm{~Hz}, 1 \mathrm{H}), 6.59$ (d, $J=$ $2.2 \mathrm{~Hz}, 1 \mathrm{H}), 5.82(\mathrm{~d}, J=2.2 \mathrm{~Hz}, 1 \mathrm{H}), 5.31(\mathrm{~s}, 2 \mathrm{H}), 4.18(\mathrm{q}, J=7.6 \mathrm{~Hz}, 2 \mathrm{H}), 1.24(\mathrm{t}, J=$ $7.7 \mathrm{~Hz}, 3 \mathrm{H}) .{ }^{13} \mathrm{C}-\mathrm{NMR}(100 \mathrm{MHz}) \delta=165.9$, 165.8, 141.2, 140.2, 137.0, 134.4, 133.4, 132.6, 130.9, 129.9, 129.2, 129.0, 128.2, 128.1, 127.7, 94.1, 65.2, 61.1, 14.0; HRMS calcd for $\mathrm{C}_{19} \mathrm{H}_{17} \mathrm{IO}_{4} 436.0171$ found 436.0172 . 


\section{Materials described in Scheme 3.}

(19) $\boldsymbol{t}$-Butyl (2-iodophenyl)carbamate: To a solution of 2-iodoaniline (15 mmol, 3.28 g) in THF (10 ml) was added a 1M solution of NaHMDS in THF (32 ml). After $15 \mathrm{~min}$, a solution of methyl chloroformate $(15 \mathrm{mmol}, 1.16 \mathrm{ml})$ in THF $(5 \mathrm{ml})$ was added and the reaction mixture was stirred for an additional $12 \mathrm{~h}$, poured into $\mathrm{NH}_{4} \mathrm{Cl}(\mathrm{aq})$, diluted with ethyl acetate $(30 \mathrm{ml})$ and the organic layer was separated. The aqueous layer was extracted two times with ethyl acetate $(20 \mathrm{ml})$ and the combined organic layers were dried over $\mathrm{MgSO}_{4}$, filtered and concentrated in vacuo. Purification by column chromatography $\left(\mathrm{SiO}_{2}\right.$ ethylacetate/hexane, 1:25, $\left.\mathrm{R}_{\mathrm{f}}=0.24\right)$ gave 4.22 of $19(88 \%)$ as a slightly yellow oil. ${ }^{1} \mathrm{H}-\mathrm{NMR}(400 \mathrm{MHz}) \delta=8.03(\mathrm{~d}, J=7.8 \mathrm{~Hz}, 1 \mathrm{H}), 7.68(\mathrm{~d}, J=7.8 \mathrm{~Hz}$, $1 \mathrm{H}), 7.26(\mathrm{t}, J=7.8 \mathrm{~Hz}, 1 \mathrm{H}), 6.81(\mathrm{~s}, 1 \mathrm{H}(\mathrm{NH})), 6.71(\mathrm{t}, J=7.8 \mathrm{~Hz}, 1 \mathrm{H}), 1.51(\mathrm{~s}, 9 \mathrm{H})$; ${ }^{13} \mathrm{C}-\mathrm{NMR}(100 \mathrm{MHz}) \delta=152.5,138.8,129.129 .1,124.6,120.1,88.7,80.7,28.1$; HRMS calcd for $\mathrm{C}_{11} \mathrm{H}_{14} \mathrm{INO}_{2} 320.0142$, found 320.0136 .

(20) Ethyl 2-[2-(bromophenyl]acrylate: A solution of (40 mmol, $9.7 \mathrm{~g})$, paraformaldehyde (333 mmol, $10 \mathrm{~g})$, TDA-1 (tris[2-(2-methoxyethoxy)ethyl]amine) (10 mmol, $3.22 \mathrm{~g}$ ) and $\mathrm{K}_{2} \mathrm{CO}_{3}(58 \mathrm{mmol}, 8 \mathrm{~g})$ in $300 \mathrm{ml}$ dry toluene was stirred in a pressure vessel $(350 \mathrm{ml})$ at $85^{\circ} \mathrm{C}$. Full conversion, indicated by TLC, was achieved after three days. The cooled solution was filtered, washed thoroughly with water, dried over $\mathrm{MgSO}_{4}$, filtered and concentrated in vacuo. Colorless oil 20, $9.99 \mathrm{~g}(78 \%)$ was obtained as pure oil and used without further purification. $\left(\mathrm{SiO}_{2}\right.$ ethylacetate $/$ hexane, $\left.1: 8, \mathrm{R}_{\mathrm{f}}=0.41\right) .{ }^{1} \mathrm{H}$ NMR $(400 \mathrm{MHz}) \delta=7.54(\mathrm{~d}, J=7.7 \mathrm{~Hz}, 1 \mathrm{H}), 7.29(\mathrm{t}, J=7.8 \mathrm{~Hz}, 1 \mathrm{H}), 7.23(\mathrm{~d}, J=7.8$ $\mathrm{Hz}, 1 \mathrm{H}), 7.17(\mathrm{t}, J=7.7 \mathrm{~Hz}, 1 \mathrm{H}), 6.49(\mathrm{~s}, 1 \mathrm{H}), 5.72(\mathrm{~s}, 1 \mathrm{H}), 4.23(\mathrm{q}, J=6.6 \mathrm{~Hz}, 2 \mathrm{H}), 1.25$ $(\mathrm{t}, J=6.6 \mathrm{~Hz}, 3 \mathrm{H}) ;{ }^{13} \mathrm{C}-\mathrm{NMR}(100 \mathrm{MHz}) \delta=165.5,142.0,138.5,132.2,130.7,129.3$, 128.5, 127.1, 123.1, 61.0, 13.9; $\mathrm{Ms}(\mathrm{EI})$ for $\mathrm{C}_{11} \mathrm{H}_{11} \mathrm{BrO}_{2} \mathrm{~m} / \mathrm{z}=175.1\left(\mathrm{M}^{+}-\mathrm{HBr}\right)$; HRMS (253.9) not possible because of instability.

\section{( 21 ) E t h y l ( 2 E ) - 2 - ( 2 - b r o m o p h e nteytt ) - 3 - \{ 2 - I}

butoxycarbonyl)aminolphenyl \}acrylate: Under argon atmosphere: To a solution of 19 $(2.96 \mathrm{mmol}, 820 \mathrm{mg})$ and $20(2.6 \mathrm{mmol}, 663 \mathrm{mg})$ in dimethylacetamide $(10 \mathrm{ml})$ was added $\mathrm{Et}_{4} \mathrm{NCl}(4 \mathrm{mmol}, 662 \mathrm{mg})$, N-methylcyclohexylamine $(6 \mathrm{mmol}, 1.54 \mathrm{ml})$ and $\mathrm{Pd}(\mathrm{OAc})_{2}(0.1 \mathrm{mmol}, 22 \mathrm{mg})$. After stirring reaction mixture for $18 \mathrm{~h}$ at $100^{\circ} \mathrm{C}$ the reaction mixture was poured into water $(10 \mathrm{ml})$ and the extracted with ethylacetate $(2 \times 20$ $\mathrm{ml}$ ). The combined organic layers were dried over $\mathrm{MgSO}_{4}$, filtered and concentrated in vacuo. Purified by column chromatography $\left(\mathrm{SiO}_{2}\right.$ ethylacetate/hexane, $\left.1: 3, \mathrm{R}_{\mathrm{f}}=0.35\right)$ to give $860 \mathrm{mg}(82 \%)$ of 21 as a colorless oil. ${ }^{1} \mathrm{H}-\mathrm{NMR}(400 \mathrm{MHz}) \delta=7.91(\mathrm{~s}, 1 \mathrm{H}), 7.77$ $(\mathrm{d}, J=7.7 \mathrm{~Hz}, 1 \mathrm{H}(\mathrm{br})), 7.55$ (d, $J=7.8 \mathrm{~Hz}, 1 \mathrm{H}), 7.20-7.09(\mathrm{~m}, 3 \mathrm{H}), 6.99(\mathrm{~d}, J=7.8 \mathrm{~Hz}$, $1 \mathrm{H}), 6.78(\mathrm{t}, J=7.8 \mathrm{~Hz}, 1 \mathrm{H}), 6.73(\mathrm{~d}, J=7.8 \mathrm{~Hz}, 1 \mathrm{H}), 6.41(\mathrm{~s},(\mathrm{NH})), 4.25(\mathrm{~m}, 2 \mathrm{H}), 1.51$ $(\mathrm{s}, 3 \mathrm{H}), 1.25(\mathrm{t}, J=6.9 \mathrm{~Hz}, 3 \mathrm{H}) ;{ }^{13} \mathrm{C}-\mathrm{NMR}(100 \mathrm{MHz}) \delta=166.3,152.6,136.8,136.5$, $135.1,132.4,131.5,129.6,129.4$, 129.3, 127.6, 124.3, 123.8, 121.6, 61.5, 52.4, 14.08; 
Ms (CI) for $\mathrm{C}_{17} \mathrm{H}_{15} \mathrm{BrO}_{3}: \mathrm{m} / \mathrm{z}=403.8\left(\mathrm{M}+\mathrm{H}^{+}\right)$; HRMS calcd for $\mathrm{C}_{20} \mathrm{H}_{20} \mathrm{BrNO}_{3}\left(\mathrm{Na}^{+}\right)$ 426.0317 found 426.0328 .

(22) Ethyl (2E)-3-(2-aminophenyl)-2-(2-bromophenyl)acrylate: To a solution of A38 $(0.72 \mathrm{mmol}, 320 \mathrm{mg})$ in DCM $(10 \mathrm{ml})$ was added TFA $(8 \mathrm{mmol}, 0.616 \mathrm{ml})$. The reaction mixture was stirred at $\mathrm{rt}$ for $14 \mathrm{~h}$, evaporate to dryness and purified by column chromatography $\left(\mathrm{SiO}_{2}\right.$ ethylacetate/hexane, 1:2, $\left.\mathrm{R}_{\mathrm{f}}=0.39\right)$ to give $251 \mathrm{mg}(99 \%)$ of 22 as a colorless oil. ${ }^{1} \mathrm{H}-\mathrm{NMR}(400 \mathrm{MHz}) \delta=7.98(\mathrm{~s}, 1 \mathrm{H}), 7.59(\mathrm{dd}, J=7.69 \mathrm{~Hz}, J=1.46 \mathrm{~Hz}$, $1 \mathrm{H}), 7.18-7.15(\mathrm{~m}, 2 \mathrm{H}), 7.08-7.05(\mathrm{~m}, 2 \mathrm{H}), 6.82(\mathrm{dd}, J=7.69 \mathrm{~Hz}, J=1.46 \mathrm{~Hz}, 1 \mathrm{H}), 6.64$ $(\mathrm{d}, J=7.69 \mathrm{~Hz}, 1 \mathrm{H}), 6.58(\mathrm{t}, J=7.69 \mathrm{~Hz}, 1 \mathrm{H}), 5.28\left(\mathrm{~m}, 2 \mathrm{H}\left(\mathrm{NH}_{2}\right)\right), 4.25(\mathrm{~m}, 2 \mathrm{H}), 1.27(\mathrm{t}$, $J=6.9 \mathrm{~Hz}, 3 \mathrm{H}) ;{ }^{13} \mathrm{C}-\mathrm{NMR}(100 \mathrm{MHz}) \delta=166.9,141.9,137.0,136.4,134.0,132.5$, 131.6, 130.2, 129.8, 129.3, 127.5, 124.6, 122.1, 120.5, 117.7, 61.5, 14.1; HRMS calcd for $\mathrm{C}_{17} \mathrm{H}_{16} \mathrm{BrNO}_{2}$ 346.0437, found 346.0429.

(23) Ethyl (2E)-2-(2-bromophenyl)-3-(28-droxyphenyl)acrylate: Under argon atmosphere: To a solution of 2-iodophenol $(3.5 \mathrm{mmol}, 770 \mathrm{mg})$ and $\mathbf{2 0}(3 \mathrm{mmol}, 765 \mathrm{mg})$ in dimethylacetamide $(10 \mathrm{ml})$ was added $\mathrm{Et}_{4} \mathrm{NCl}(4 \mathrm{mmol}, 662 \mathrm{mg})$, Nmethylcyclohexylamine $(6 \mathrm{mmol}, 1.54 \mathrm{ml})$ and $\mathrm{Pd}(\mathrm{OAc})_{2}(0.1 \mathrm{mmol}, 22 \mathrm{mg})$. After stirring reaction mixture for $18 \mathrm{~h}$ at $100^{\circ} \mathrm{C}$ the reaction mixture was poured into water $(10$ $\mathrm{ml})$ and the extracted with ethylacetate $(2 \times 20 \mathrm{ml})$. The combined organic layers were dried over $\mathrm{MgSO}_{4}$, filtered and concentrated in vacuo. Purified by column chromatography $\left(\mathrm{SiO}_{2}\right.$ ethylacetate/hexane, 1:3, $\left.\mathrm{R}_{\mathrm{f}}=0.25\right)$ to give $850 \mathrm{mg}(82 \%)$ of 22 as a colorless oil. ${ }^{1} \mathrm{H}-\mathrm{NMR}(400 \mathrm{MHz}) \delta=8.27(\mathrm{~s}, 1 \mathrm{H}), 7.63(\mathrm{dd}, J=7.7 \mathrm{~Hz}, J=1.9 \mathrm{~Hz}$, $1 \mathrm{H}), 7.25-7.17(\mathrm{~m}, 2 \mathrm{H}), 7.11-7.05(\mathrm{~m}, 2 \mathrm{H}), 6.77(\mathrm{dd}, J=7.7 \mathrm{~Hz}, J=1.9 \mathrm{~Hz}, 1 \mathrm{H}), 6.61$ $(\mathrm{m}, 2 \mathrm{H}), 5.74(\mathrm{bs}, \mathrm{OH}, 1 \mathrm{H}), 4.28(\mathrm{~m}, 2 \mathrm{H}), 1.27(\mathrm{t}, J=6.9 \mathrm{~Hz}, 3 \mathrm{H}) ;{ }^{13} \mathrm{C}-\mathrm{NMR}(100 \mathrm{MHz})$ $\delta=167.8,155.6,137.4,135.5,132.5,131.5,131.3,130.7,129.6,129.3,127.6,124.6$, 121.4, 119.7, 115.8, 61.1, 13.9; Ms (CI) for $\mathrm{C}_{17} \mathrm{H}_{15} \mathrm{BrO}_{3}: \mathrm{m} / \mathrm{z}=347.0\left(\mathrm{M}+\mathrm{H}^{+}\right)$; HRMS calcd for $\mathrm{C}_{17} \mathrm{H}_{15} \mathrm{BrO}_{3}, 347.0283$ found 347.0292 .

(24) Ethyl 5H-dibenzo[b,f]azepine-10-carboxylate: Under argon atmosphere: A solution of $\mathbf{A 3 9}(0.64 \mathrm{mmol}, 220 \mathrm{mg}), \mathrm{K}_{3} \mathrm{PO}_{4}(1.3 \mathrm{mmol}, 275 \mathrm{mg})$ and $\mathrm{Pd}\left(t-\mathrm{Bu}_{3} \mathrm{P}\right)_{2}(0.032$ $\mathrm{mmol}, 16.3 \mathrm{mg})$ in toluene $(10 \mathrm{ml})$ was heated for $16 \mathrm{~h}$ at $90^{\circ} \mathrm{C}$. The reaction mixture was poured into water $(10 \mathrm{ml})$ and the extracted with ethylacetate $(2 \times 20 \mathrm{ml})$. The combined organic layers were dried over $\mathrm{MgSO}_{4}$, filtered and concentrated in vacuo. Purification by column chromatography $\left(\mathrm{SiO}_{2}\right.$ ethylacetate/hexane, $\left.1: 3, \mathrm{R}_{\mathrm{f}}=0.40\right)$ give $125 \mathrm{mg}(74 \%)$ of 23 as a red oil. ${ }^{1} \mathrm{H}-\mathrm{NMR}(400 \mathrm{MHz}) \delta=7.72(\mathrm{~s}, 1 \mathrm{H}), 7.24(\mathrm{~d}, J=7.8 \mathrm{~Hz}, 1 \mathrm{H}), 7.17-7.13$ $(\mathrm{m}, 3 \mathrm{H}), 6.99$ (t, $J=7.8 \mathrm{~Hz}, 1 \mathrm{H}), 6.92(\mathrm{t}, J=7.8 \mathrm{~Hz}, 1 \mathrm{H}), 6.71-6.66(\mathrm{~m}, 2 \mathrm{H}), 5.15$ (s, $(\mathrm{NH})), 4.33(\mathrm{~m}, 2 \mathrm{H}), 1.35(\mathrm{t}, J=6.8 \mathrm{~Hz}, 3 \mathrm{H}) ;{ }^{13} \mathrm{C}-\mathrm{NMR}(100 \mathrm{MHz}) \delta=167.6,150.6$, 150.2 , 140.8, 133.6, 131.8, 130.9, 130.8, 129.5, 127.8, 127.7, 123.4, 123.2, 120.7, 119.5, 61.1, 14.2; HRMS calcd for $\mathrm{C}_{17} \mathrm{H}_{15} \mathrm{NO}_{2} 266.1176$, found 266.1178.

(25) Ethyllibenzo[b,f]oxepine-10-carboxylate: Under argon atmosphere: To a mixture of $\mathrm{K}_{3} \mathrm{PO}_{4}(1.61 \mathrm{mmol}, 341 \mathrm{mg}), \mathrm{Pd}(\mathrm{OAc})_{2}(0.081 \mathrm{mmol}, 18 \mathrm{mg})$ and 2-(di-tertbutylphosphino)biphenyl $(0.121 \mathrm{mmol}, 36 \mathrm{mg})$ was added a solution of $23(0.8 \mathrm{mmol}$, 
$280 \mathrm{mg})$ in toluene $(3 \mathrm{ml})$. The reaction mixture was stirred at $100^{\circ} \mathrm{C}$ for $14 \mathrm{~h}$ and was poured into water $(10 \mathrm{ml})$ and the extracted with ethylacetate $(2 \times 20 \mathrm{ml})$. The combined organic layers were dried over $\mathrm{MgSO}_{4}$, filtered and concentrated in vacuo. Purified by column chromatography $\left(\mathrm{SiO}_{2}\right.$ ethylacetate/hexane, 1:6, $\left.\mathrm{R}_{\mathrm{f}}=0.64\right)$ give $129 \mathrm{mg}(60 \%)$ of 24 as a colorless oil. ${ }^{1} \mathrm{H}-\mathrm{NMR}(400 \mathrm{MHz}) \delta=7.90(\mathrm{~s}, 1 \mathrm{H}), 7.52(\mathrm{dd}, J=7.7 \mathrm{~Hz}, J=1.9$ $\mathrm{Hz}, 1 \mathrm{H}), 7.38-7.30(\mathrm{~m}, 3 \mathrm{H}), 7.22(\mathrm{~m}, 2 \mathrm{H}), 7.16(\mathrm{~m}, 2 \mathrm{H}), 4.37(\mathrm{q}, J=7.2 \mathrm{~Hz}, 2 \mathrm{H}), 1.38(\mathrm{t}$, $J=7.2 \mathrm{~Hz}, 3 \mathrm{H}) ;{ }^{13} \mathrm{C}-\mathrm{NMR}(100 \mathrm{MHz}) \delta=166.8,159.0,158.8,137.7,131.6,131.5,130.7$, $130.2,128.8,127.7,124.9,124.6,121.3,121.1,61.3,14.9$; HRMS calcd for $\mathrm{C}_{17} \mathrm{H}_{14} \mathrm{O}_{3}$ (M+Li) 273.1103, found 273.1103. 


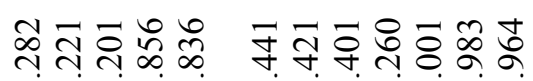

ring

(1) 2,2,2-Trifluoro-N-(2-iodo-phenyl)-acetamide

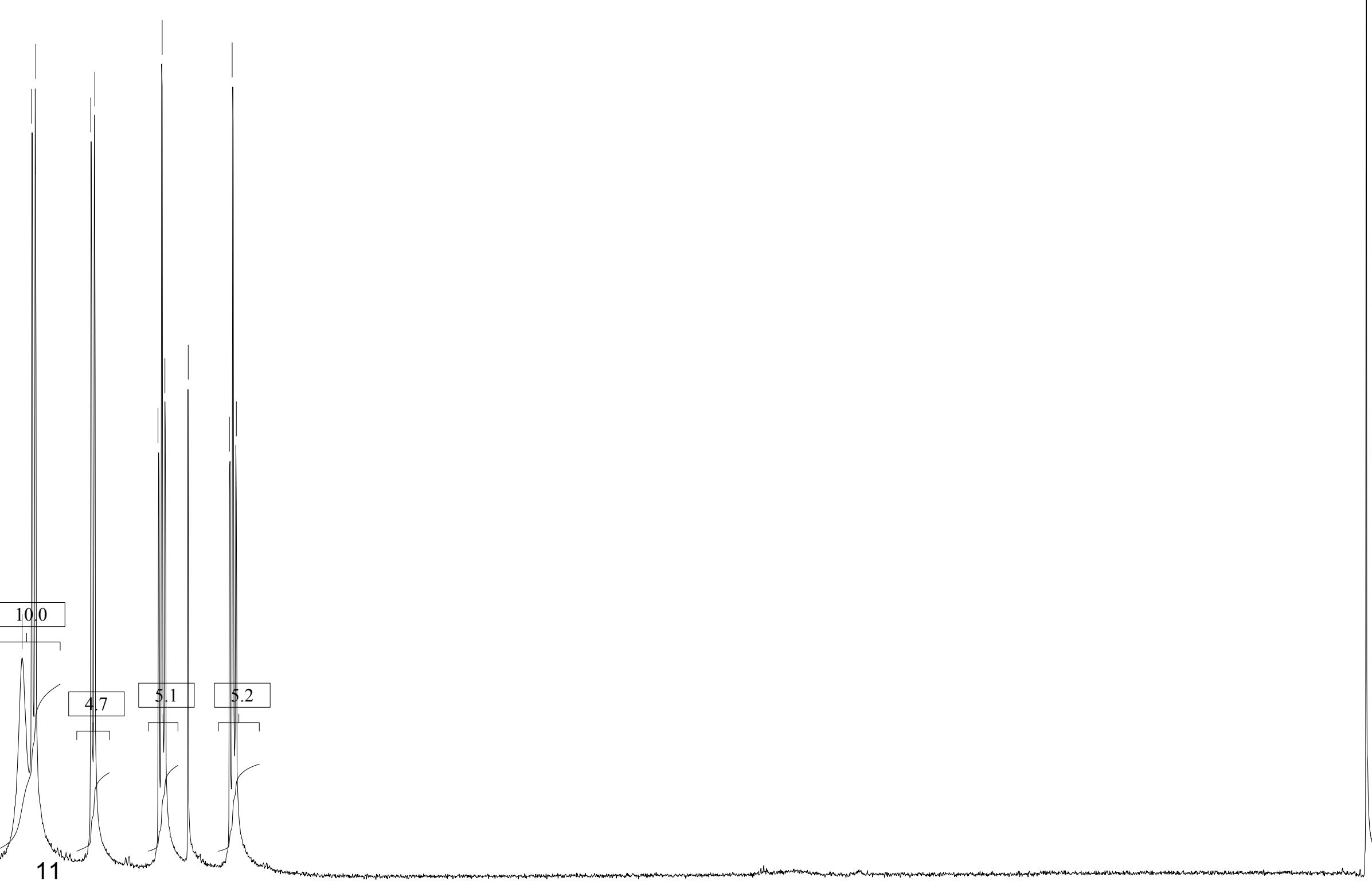

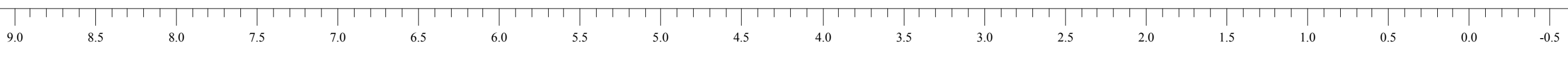




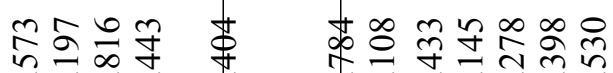

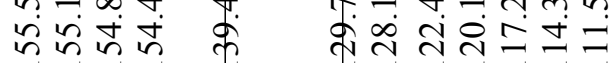

तु.

(1) 2,2,2-Trifluoro-N-(2-iodo-phenyl)-acetamide 


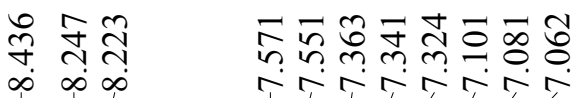

(2) 2,2,2-Trifluoro-N-(2-bromo-phenyl)acetamide 
(2) 2,2,2-Trifluoro-N-(2-bromo-phenyl)acetamide 
(5) Ethyl 2-(2-\{[(2-iodo-phenyl)-(2,2,2-trifluoro-acetyl)-amino]-methyl\}-phenyl)-acrylate 


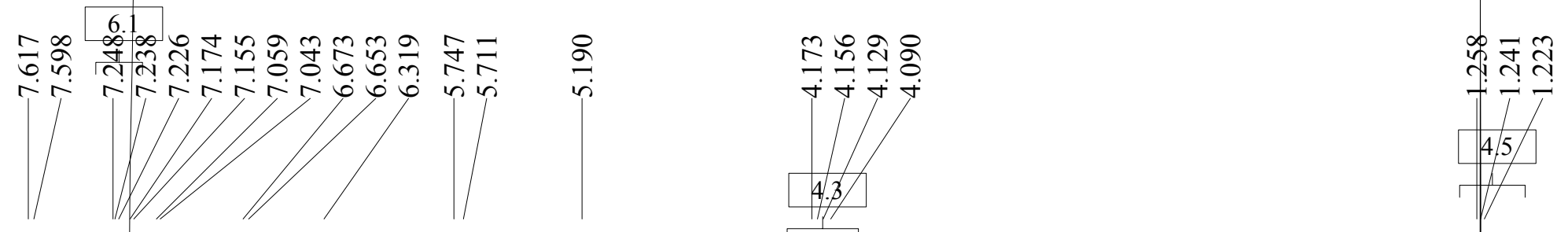

(6) Ethyl 2-(2-\{[(2-bromo-phenyl)-(2,2,2-trifluoro-acetyl)-amino]-methyl\}-phenyl)-acrylate 


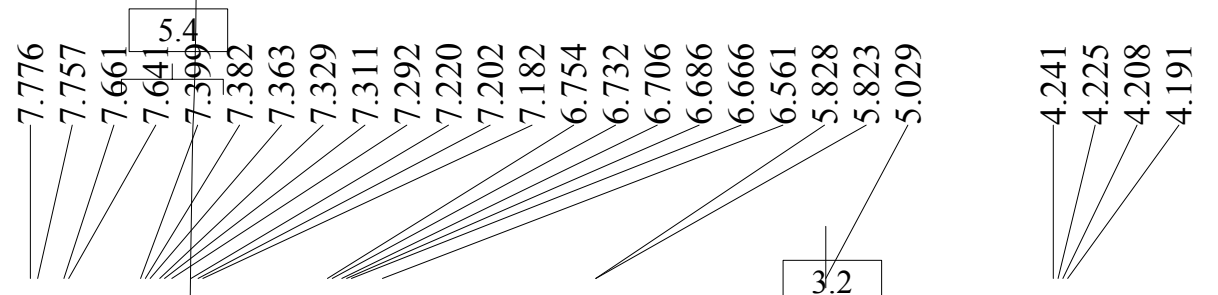

(7) Ethyl 2-\{2-[(2-iodophenoxy)methyl]phenyl\}acrylate

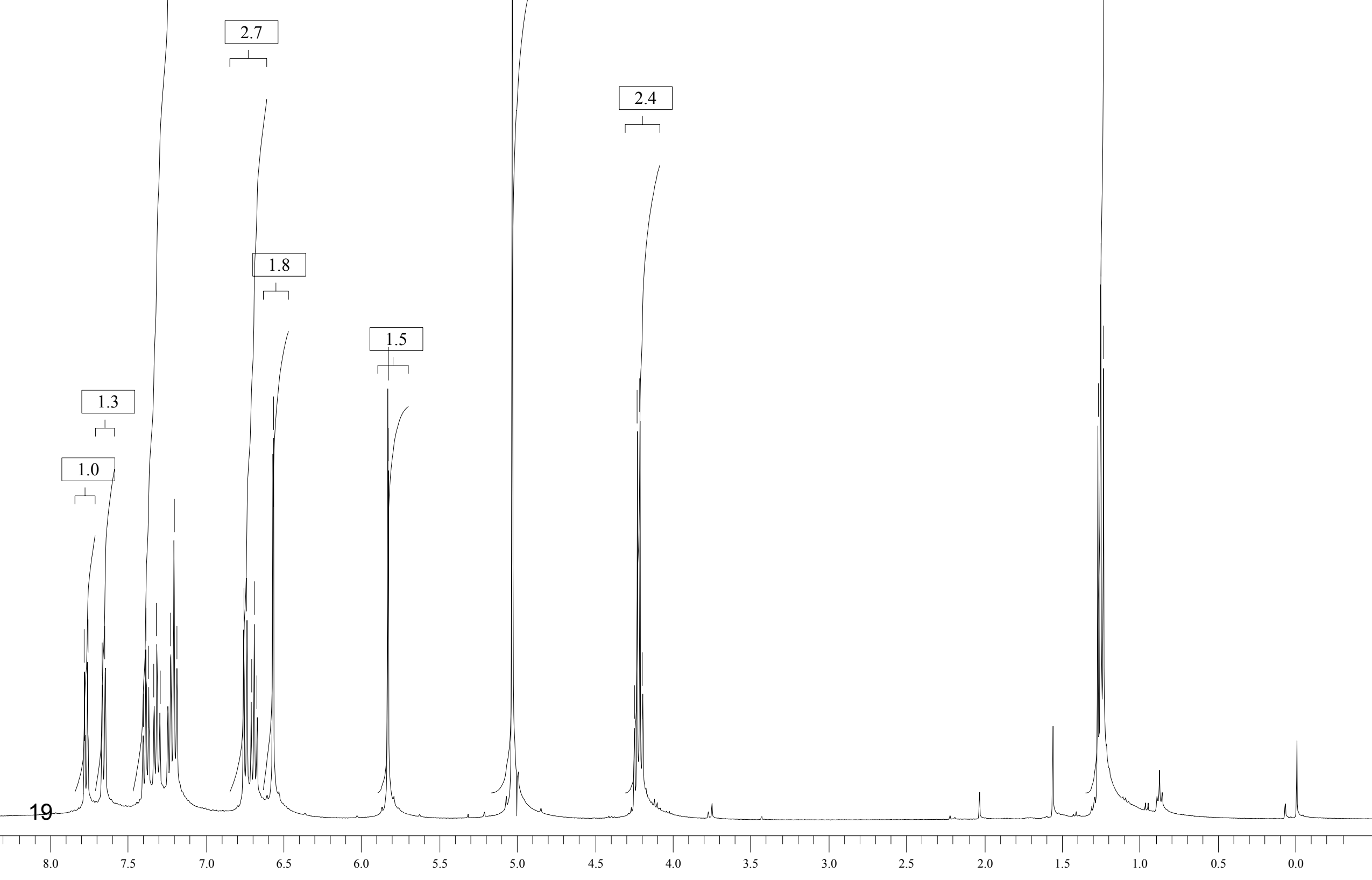


(8) Ethyl 2-\{2-[(2-bromophenoxy)methyl]phenyl\}acrylate
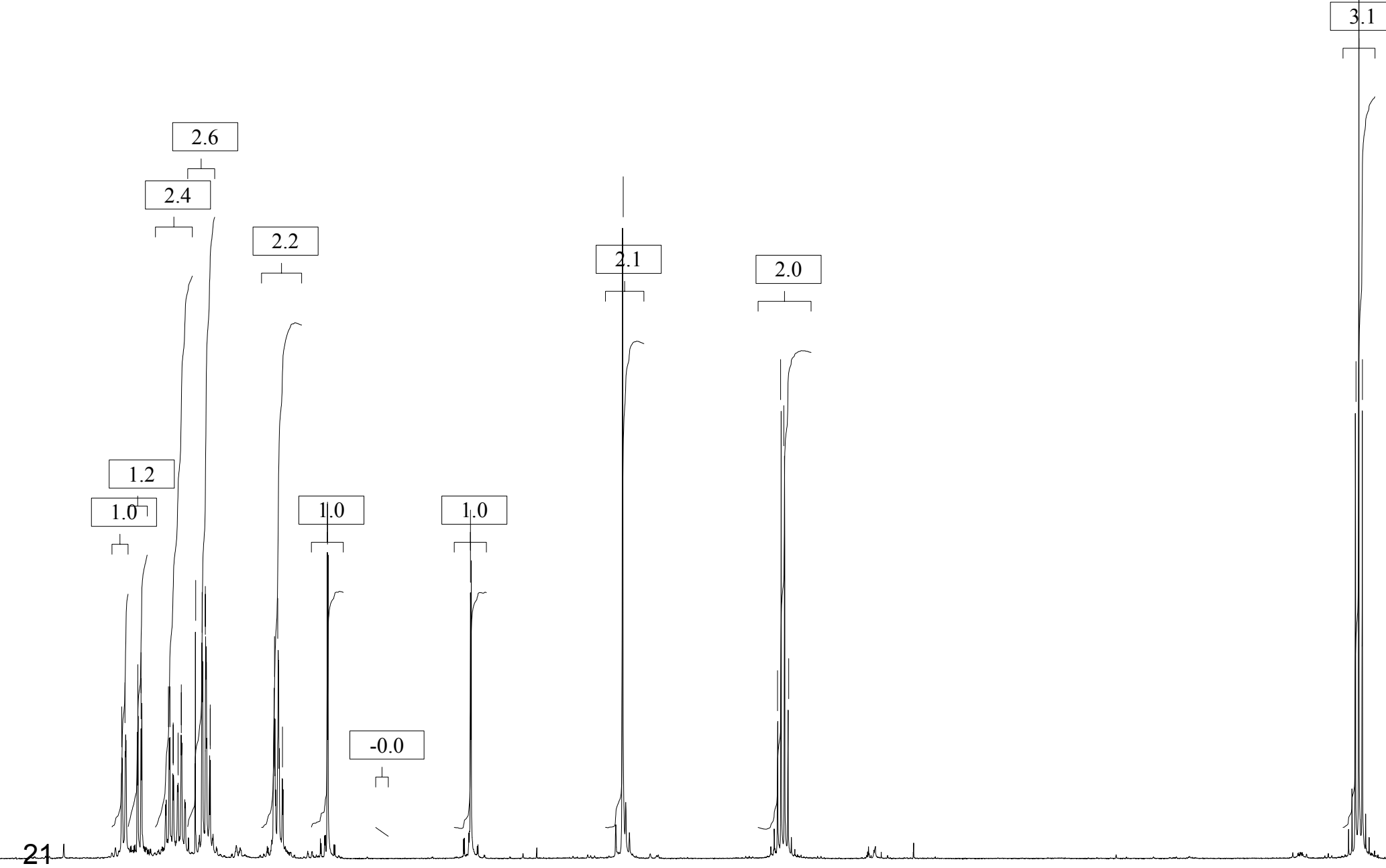


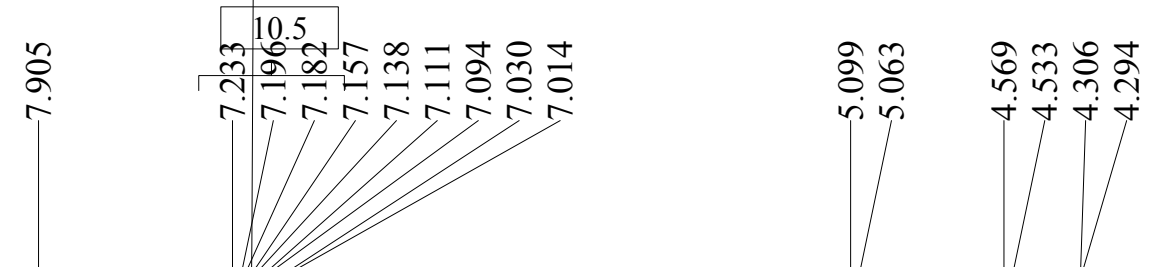

(9) Ethyl 5-(trifluoroacetyl)-5,6-dihydrodibenzo[b,f]azocine-11-carboxylate 
(9) Ethyl 5-(trifluoroacetyl)-5,6-dihydrodibenzo[b,f]azocine-11-carboxylate 


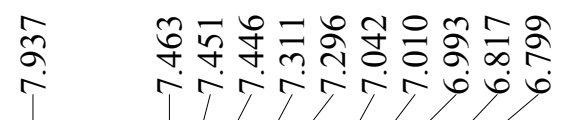

$\stackrel{+}{m}$

잉ำ

ले ๗ิำ

is

(10) Ethyl 6H-dibenzo[b,f]oxocine-11-carboxylate

3.6

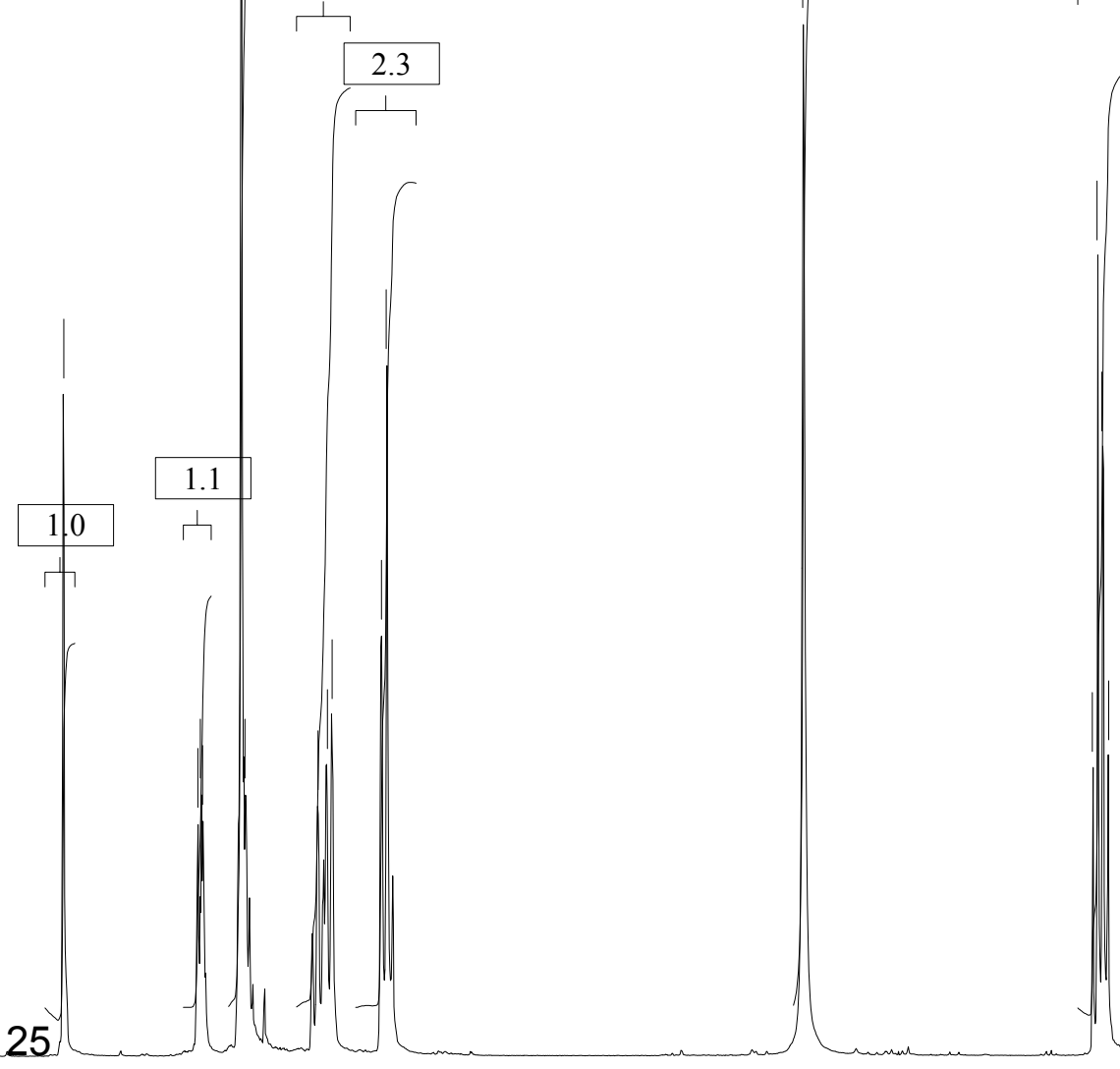




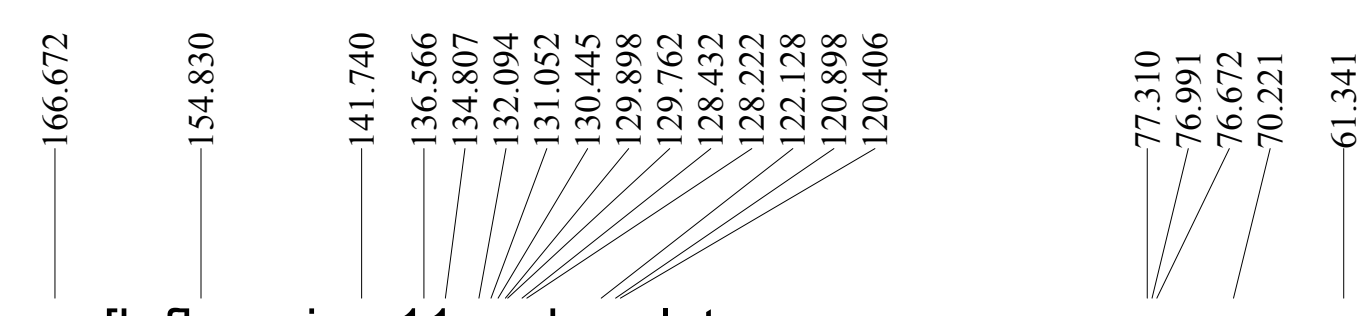

(10) Ethyl 6H-dibenzo[b,f]oxocine-11-carboxylate 
(11) Ethyl 2-[2-(bromomethyl)phenyl]acrylate 
(5) Ethyl 2-(2-\{[(2-iodo-phenyl)-(2,2,2-trifluoro-acetyl)-amino]-methyl\}-phenyl)-acrylate 


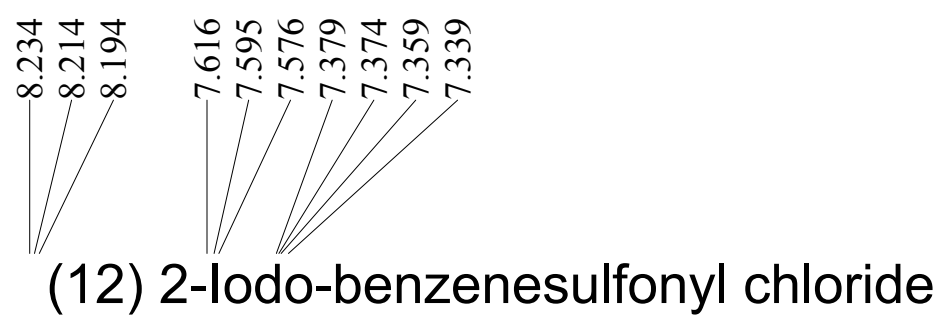

(12) 2-lodo-benzenesulfonyl chloride

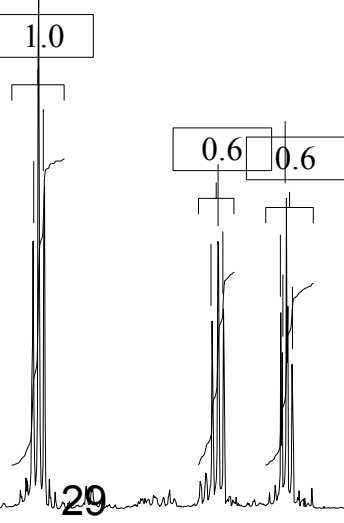




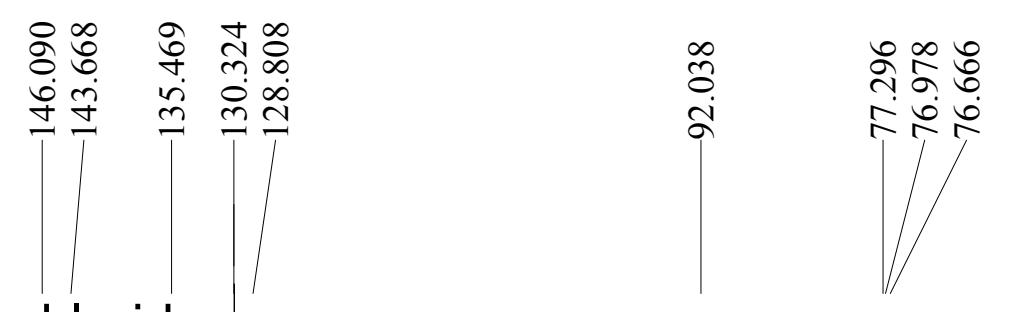

(12) 2-lodo-benzenesulfonyl chloride 


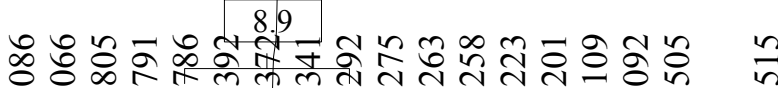
क

(14) Ethyl 2-(2-\{[(2-iodophenyl)sulfonyl]methyl\}phenyl)acrylate 
(14) Ethyl 2-(2-\{[(2-iodophenyl)sulfonyl]methyl\}phenyl)acrylate 
(15) Ethyl 2-(2-\{[(2-bromophenyl)sulfonyl]methyl\}phenyl)acrylate

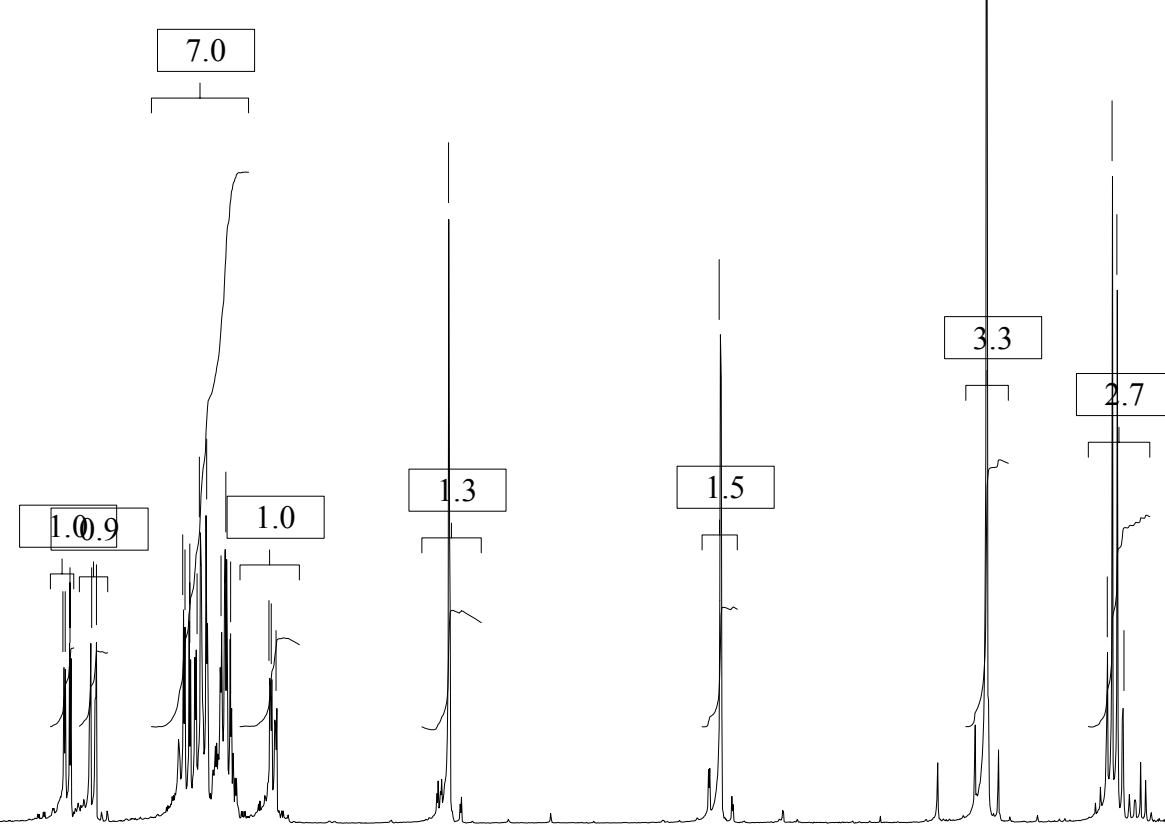


(15) Ethyl 2-(2-\{[(2-bromophenyl)sulfonyl]methyl\}phenyl)acrylate 


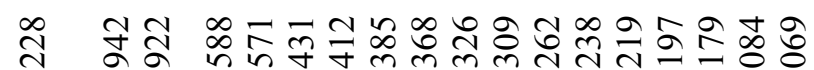

(16) Ethyl 6H-dibenzo[b,f]thiocine-11-carboxylate 5,5-dioxide

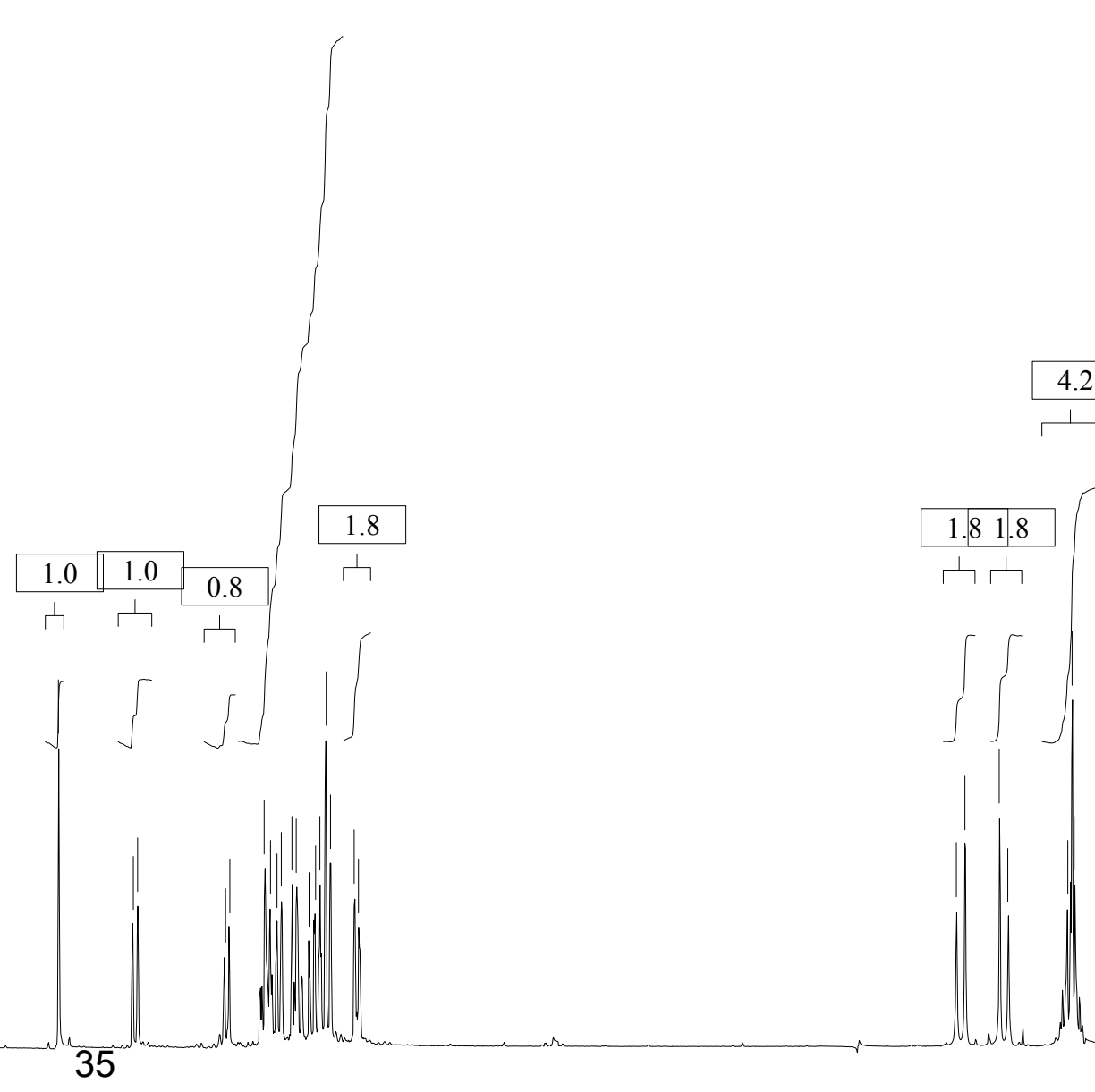




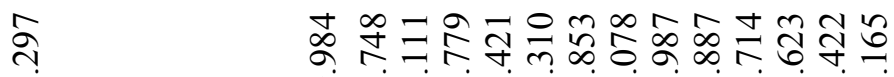

(16) Ethyl 6H-dibenzo[b,f]thiocine-11-carboxylate 5,5-dioxide 


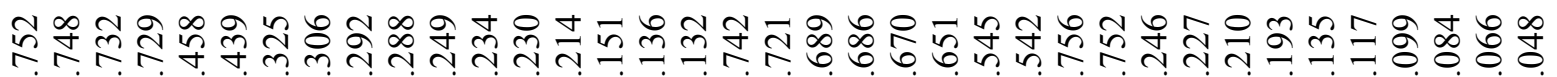

(17) Ethyl 2-\{2-[2-(2-iodophenoxy)ethyl]phenyl\}acrylate

2.0

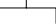

$\mapsto \longdiv { 0 . 8 }$ 


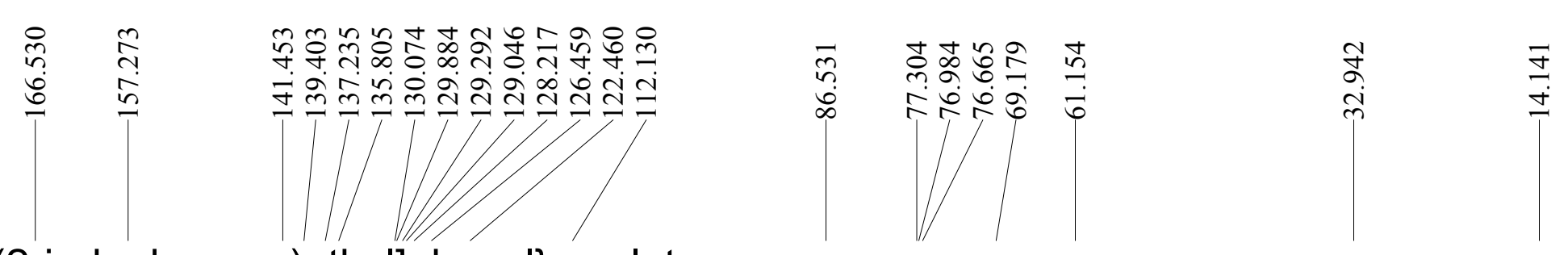

(17) Ethyl 2-\{2-[2-(2-iodophenoxy)ethyl]phenyl\}acrylate 38 


\section{(18) 2-[1-(Ethoxycarbonyl)vinyl]benzyl 2-iodobenzoate}


(18) 2-[1-(Ethoxycarbonyl)vinyl]benzyl 2-iodobenzoate

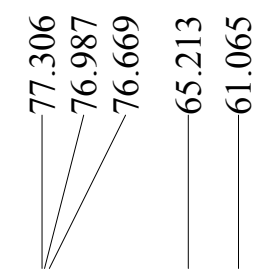




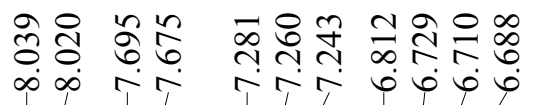

(19) t-Butyl (2-iodophenyl)carbamate 
(19) t-Butyl (2-iodophenyl)carbamate 
(20) Ethyl 2-[2-(bromophenyl]acrylate 
\%े 苟宫

(20) Ethyl 2-[2-(bromophenyl]acrylate 


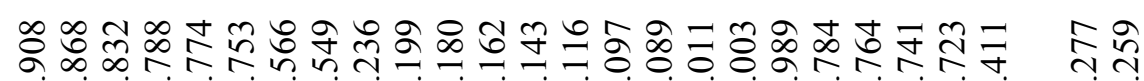

rinining

(21) Ethyl (2E)-2-(2-bromophenyl)-3-\{2-[(tert-butoxycarbonyl)amino]phenyl\}acrylate

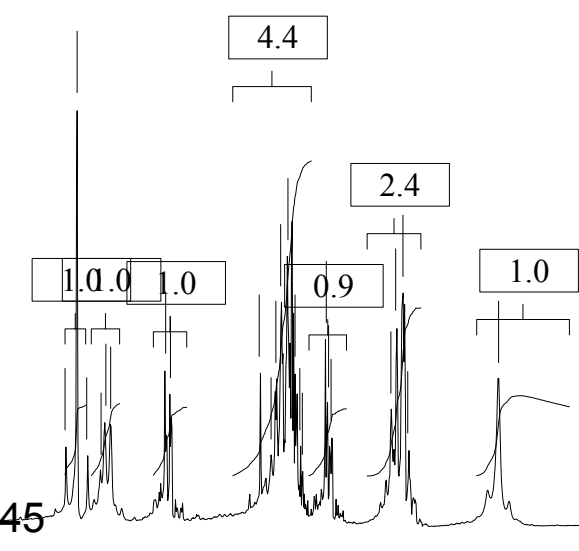


(21) Ethyl (2E)-2-(2-bromophenyl)-3-\{2-[(tert-butoxycarbonyl)amino]phenyl\}acrylate 


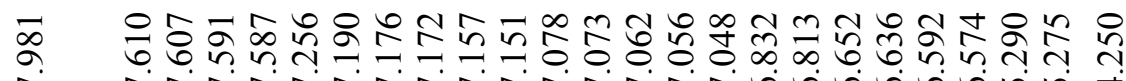

$\rightarrow$ in

(22) Ethyl (2E)-3-(2-aminophenyl)-2-(2-bromophenyl)acrylate 
(23) Ethyl (2E)-2-(2-bromophenyl)-3-(2-hydroxyphenyl)acrylate

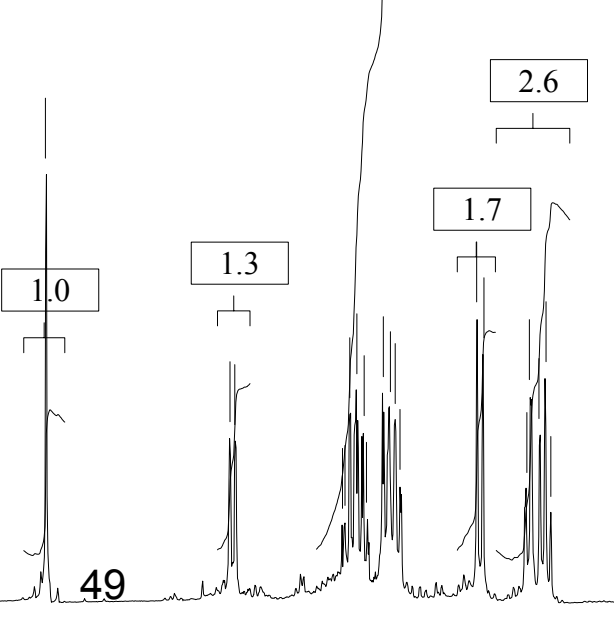




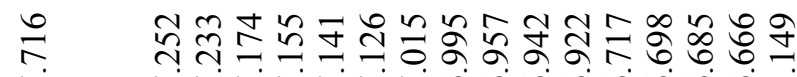

(24) Ethyl 5H-dibenzo[b,f]azepine-10-carboxylate 

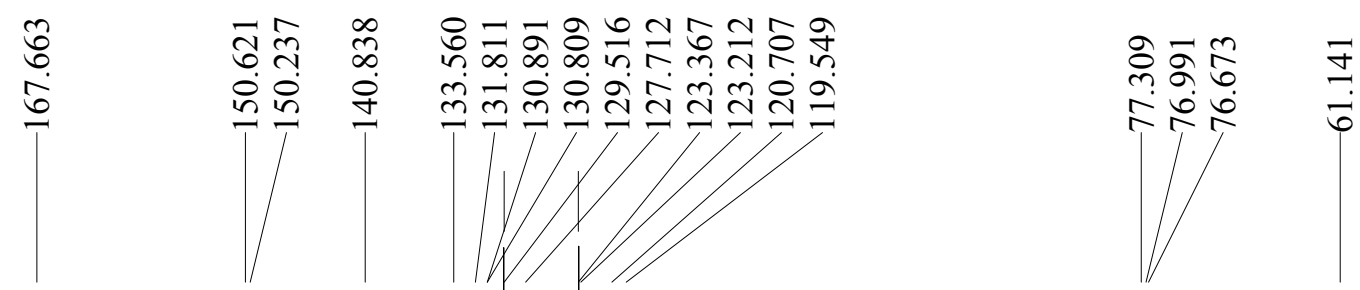

(24) Ethyl 5H-dibenzo[b,f]azepine-10-carboxylate 


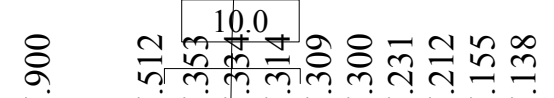

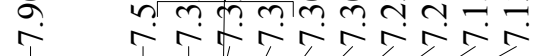

(25) Ethyl dibenzo[b,f]oxepine-10-carboxylate 
(S1) Ethyl (2-methylphenyl)acetate 


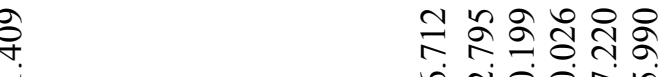

(S1) Ethyl (2-methylphenyl)acetate 

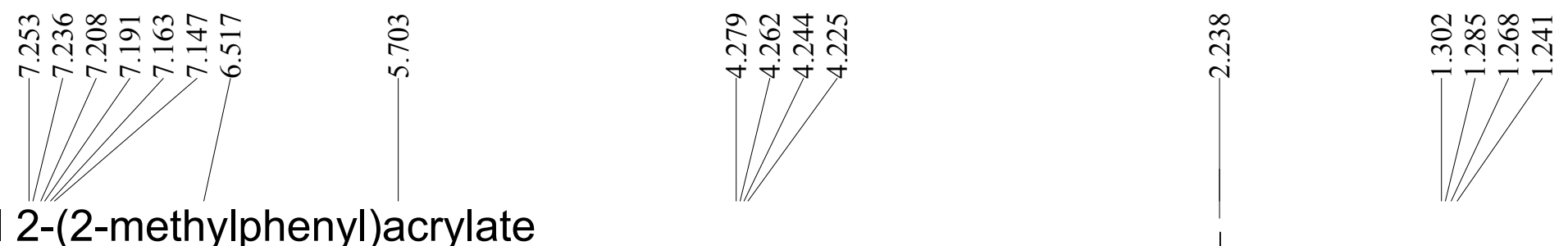

(S2) Ethyl 2-(2-methylphenyl)acrylate 
(S3) 2-[2-(2-Bromophenyl)ethoxy]tetrahydro-2H-pyran

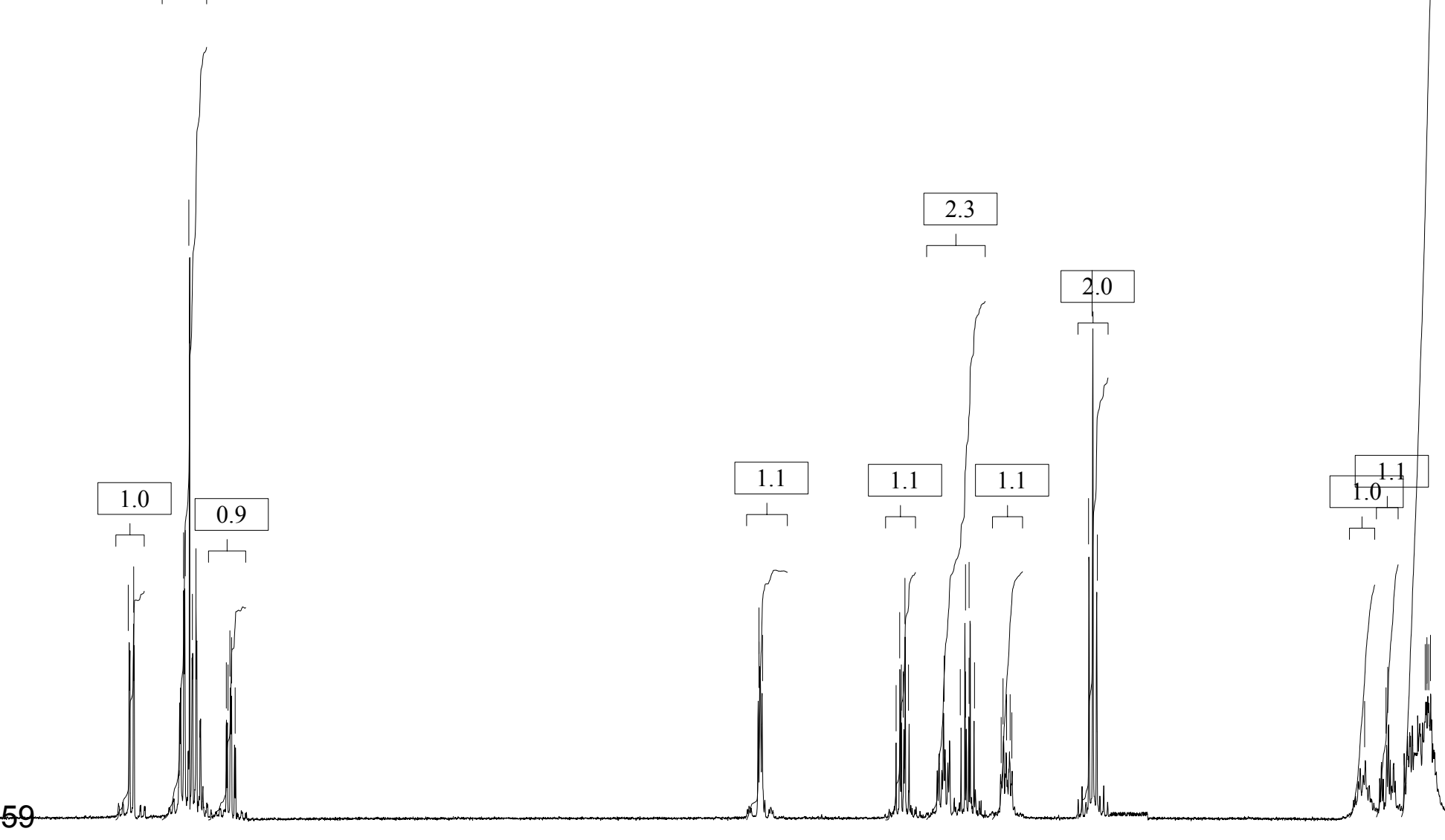


(S3) 2-[2-(2-Bromophenyl)ethoxy]tetrahydro-2H-pyran 
(S4) Ethyl 2-[2-(2-hydroxyethyl)phenyl]acrylate 
(S4) Ethyl 2-[2-(2-hydroxyethyl)phenyl]acrylate 